\title{
Tomato Stress-Associated Protein 4 Contributes Positively to Immunity Against Necrotrophic Fungus Botrytis cinerea
}

\author{
Shixia Liu, Xi Yuan, Yuyan Wang, Hui Wang, Jiali Wang, Zhihui Shen, Yizhou Gao, Jiating Cai, \\ Dayong $\mathrm{Li}^{\dagger}$, and Fengming Song ${ }^{\dagger}$
}

National Key Laboratory for Rice Biology and Key Laboratory of Crop Diseases and Insect Pests of Ministry of Agriculture, Institute of Biotechnology, Zhejiang University, Hangzhou 310058, P. R. China

Accepted 8 November 2018.

\begin{abstract}
Stress-associated proteins (SAPs) are A20 and AN1 domaincontaining proteins, some of which play important roles in plant stress signaling. Here, we report the involvement of tomato $S I S A P$ family in immunity. SISAPs responded with different expression patterns to Botrytis cinerea and defense signaling hormones. Virus-induced gene silencing of each of the SISAP genes and disease assays revealed that SlSAP4 and $S I S A P 10$ play roles in immunity against $B$. cinerea. Silencing of $S I S A P 4$ resulted in attenuated immunity to $B$. cinerea, accompanying increased accumulation of reactive oxygen species and downregulated expression of jasmonate and ethylene (JA/ET) signaling-responsive defense genes. Transient expression of SISAP4 in Nicotiana benthamiana led to enhanced resistance to $B$. cinerea. Exogenous application of methyl jasmonate partially restored the resistance of the SISAP4silenced plants against $B$. cinerea. SISAP4 interacted with three of four SIRAD23 proteins. The A20 domain in SISAP4 and the Ub-associated domains in SIRAD23d are critical for SISAP4-SIRAD23d interaction. Silencing of SIRAD23d led to decreased resistance to $B$. cinerea, but silencing of each of other $S I R A D 23 s$ did not affect immunity against $B$. cinerea. Furthermore, silencing of SISAP4 and each of the SIRAD23s did not affect immunity to Pseudomonas syringae pv. tomato DC3000. These data suggest that SISAP4 contributes positively to tomato immunity against $B$. cinereal through affecting JA/ET signaling and may be involved in the substrate ubiquitination process via interacting with SIRAD23d.
\end{abstract}

Plant fungal diseases represent a worldwide threat to food security and ecosystem health (Dean et al. 2012). Based on their lifestyle, plant-pathogenic fungi have been classified as biotrophs that feed on living hosts, hemibiotrophs that

${ }^{\dagger}$ Corresponding authors: F. Song: fmsong@zju.edu.cn and D. Li: dyli@zju.edu.cn

Funding: This study was supported by Zhejiang Provincial National Science Foundation of China (grant number LY16C140001) and the National Key Research and Development Program of China (grant number 2016YFD0100600).

*The $\boldsymbol{e}$-Xtra stands for "electronic extra" and indicates that four supplementary figures and two supplementary tables are published online.

() 2019 The American Phytopathological Society possess a biotrophic phase during the early stage of infection, and necrotrophs that actively kill hosts to acquire nutrients (Wang et al. 2014). To combat pathogenic attack, plants have evolved to possess two distinct types of innate immune responses, which are referred to as pathogen-, microbe-, or damage-associated molecular pattern (PAMP, MAMP, or DAMP)-triggered immunity (PTI) and effector-triggered immunity (ETI) (Jones and Dangl 2006; Boller and He 2009; Spoel and Dong 2012). PTI is generally triggered by plant recognition of different PAMPs, MAMPs, or DAMPs, forming the first layer of the innate immune responses, whereas ETI is specifically triggered by the interactions between plant resistance $(\mathrm{R})$ proteins and pathogen avirulence proteins, developing as a specialized form of the innate immune responses (Bigeard et al. 2015; Cui et al. 2015; Schwessinger and Ronald 2012; Segonzac and Zipfel 2011; Zhang and Zhou 2010). Whereas both PTI and ETI are required for resistance to biotrophs and hemibiotrophs, PTI is believed to be an active resistance response against necrotrophs (Lai and Mengiste 2013; Mengiste 2012). However, ETI may also play a role in immunity against necrotrophs, although the effector that triggers ETI is not yet known (Wang et al. 2014).

Upon perception of pathogen-derived signals, plants often activate a network of defense hormone-mediated signaling pathways (Peng et al. 2018; Pieterse et al. 2009; Zhang et al. 2017), which ultimately lead to transcriptional reprogramming that coordinately regulates expression of a large set of genes $(\mathrm{Li}$ et al. 2016; Tsuda and Somssich 2015). Phytohormones such as salicylic acid (SA), jasmonates (JA), and ethylene (ET) are critical defense signaling molecules, forming the SA signaling and the JA/ET signaling pathways, respectively (Broekaert et al. 2006; Pieterse et al. 2012; Shigenaga and Argueso 2016). Generally, JA/ET signaling positively regulates immunity against necrotrophs, whereas SA signaling positively modulates immunity against biotrophs and hemibiotrophs (Glazebrook 2005). Mutually antagonistic or synergistic crosstalks between SA and JA/ET signaling often provide priority for plants to activate, timely and strongly, the defense response against the invading pathogens (Derksen et al. 2013).

Stress-associated proteins (SAPs) are A20 and AN1 domain-containing zinc-finger proteins and have been shown to act as key regulators of innate immunity in human (Beyaert et al. 2000). Plant homologs of SAPs have been characterized in a variety of plant species and consist of a gene family with 14, 18, and 13 members in Arabidopsis, rice, and tomato, respectively (Solanke et al. 2009; Ströher et al. 2009; Vij and Tyagi 2006). Functional studies have suggested that several 
SAPs in Arabidopsis, rice, and other plant species play important roles in modulating abiotic stress tolerance (Giri et al. 2013). Meanwhile, the involvement of SAPs in plant immunity has recently been discovered. It was found that the rice OsSAP genes showed responsiveness toward biotic stress simulators such as elicitors, defense signaling molecules, and pathogens and that constitutive expression of OsSAPl in transgenic tobacco resulted in enhanced resistance against a virulent bacterial pathogen, Pseudomonas syringae pv. tomato DC3000, together with the upregulation of known defense genes (Tyagi et al. 2014). Similarly, expression of the Arabidopsis AtSAP9 was up-regulated by nonhost and host bacterial pathogens $P$. syringae pv. tabaci and $P$. syringae $\mathrm{pv}$. maculicola, respectively, and that overexpression of AtSAP9 led to increased susceptibility to infection by nonhost pathogen $P$. syringae pv. phaseolicola, indicating a negative role of AtSAP9 in immunity (Kang et al. 2017). More recently, it was found that orchid A20/AN1 protein Pha13 and its Arabidopsis homolog AtSAP5 serve as the important hub to mediate antiviral immunity (Chang et al. 2018).

At the biochemical level, some SAPs act as ubiquitin E3 ligases and link the substrate ubiquitination process with proteasomal degradation events. For examples, the Arabidopsis AtSAP5 and AtSAP9 and rice OsSAP7 possess ubiquitin E3 ligase activity in vitro and function as negative regulators of stress signaling by targeting their substrates for degradation (Kang et al. 2011, 2013, 2017; Sharma et al. 2015). Alternatively, some SAPs exert their biological functions through interacting with other proteins involved in the ubiquitination pathway. The Arabidopsis AtSAP5 recognizes ubiquitin and binds polyubiquitin rather than monoubiquitin, whereas Prunus PpSAP1 strongly interacts with polyubiquitinated proteins (Lloret et al. 2017). AtSAP9 was found to interact with RADIATION SENSITIVE23d (RAD23d) (Kang et al. 2017), belonging to the RAD23 family that acts as principal shuttles of ubiquitin conjugates (Guzder et al. 1998; Shuck et al. 2008). Arabidopsis AtRAD23s have been shown to be involved in the transfer of ubiquitinated proteins to the ubiquitin/26S proteasome system (UPS) (Farmer et al. 2010). It was thus speculated that AtSAP9 may mediate the interaction of ubiquitinated targets with UPS via AtRAD23d (Kang et al. 2017).

Botrytis cinerea, causing gray mold disease, is a typical necrotrophic fungus that infects almost all vegetable and fruit crops (Wang et al. 2014). Significant advances in understanding of the genetic and molecular basis for immunity against B. cinerea have recently been made in Arabidopsis and tomato (AbuQamar et al. 2017; Lai and Mengiste 2013; Mengiste 2012). Knowledge on the mechanism of host immune responses is most relevant to future development of novel strategies for disease control. Previous studies have identified a total of 13 SISAP genes in tomato and were found to be responsive to a variety of abiotic stresses (Solanke et al. 2009). The present study investigated, combining a virus-induced gene silencing (VIGS) approach with disease phenotyping assays, the possible involvement of SISAPs in tomato immunity against $B$. cinerea. Our results showed that silencing of either SISAP4 or SISAP1O led to decreased resistance while transient expression of SISAP4 in Nicotiana benthamiana conferred enhanced resistance against $B$. cinerea. In SlSAP4-silencing plants, $B$. cinerea-induced JA and expression of JA/ET signaling defense genes were suppressed, while exogenous application of methyl jasmonate (MeJA) partially restored the resistance to B. cinerea. SISAP4 interacted with three of four SIRAD23 proteins and silencing of SIRAD23d also led to decreased resistance against $B$. cinerea. These data demonstrate that SISAP4 contributes positively to immunity against $B$. cinerea through affecting the JA/ET signaling and may be involved in linking the substrate ubiquitination process with UPS via interacting with SIRAD23d.

\section{RESULTS}

\section{Expression patterns of SISAPs in response} to $B$. cinerea and defense signaling hormones.

We first analyzed the responsiveness of SISAP genes to $B$. cinerea and defense signaling hormones. In $B$. cinereainoculated plants, the expression levels of the SISAP genes, except that of SISAP9, were remarkably induced by two to fivefold over those in the mock-inoculated plants, at $24 \mathrm{~h}$ postinoculation (hpi) (Fig. 1A). The expression levels of SlSAP1, SlSAP3, SlSAP4, SlSAP6, SlSAP7, SISAP11, and SISAP12 were increased at 24 hpi. Especially, the expression levels of SISAP3 and SISAP6 were increased even at an earlier time (12 hpi) (Fig. 1A). In SA-treated plants, the expression levels of SISAP5, SlSAP6, SlSAP7, SISAP8, SISAP9, SISAP10, SISAP11, SISAP12, and SISAP13 were significantly induced by 2.5 - to 5.3 -fold at $48 \mathrm{~h}$ posttreatment (hpt) (Fig. 1B). MeJA treatment induced the expression of SISAP1, SISAP2, and SISAP6 by 4.2-, 3.4-, and 3.5-fold, respectively, at $48 \mathrm{hpt}$ (Fig. 1B). The expression of the SlSAP genes, with the exception of $S I S A P 2, S I S A P 3$, and SISAP13, was dramatically induced by 1-aminocyclopropane-1-carboxylicacid (ACC) (a precursor of ET), showing 2.3- to 6.0-fold of induction at $48 \mathrm{hpt}$ (Fig. 1B). Particularly, B. cinerea and ACC induced significantly the expression of SlSAP4 by 2.4-fold at 24 hpi and 5.6fold at $48 \mathrm{hpt}$, respectively (Fig. 1A and B); however, SA and MeJA did not significantly affect the expression of SISAP4 during the experiment period (Fig. 1B). These data indicate that the SISAP genes responded with different expression patterns to $B$. cinerea and defense signaling hormones.

\section{Silencing of $S I S A P 4$ resulted in attenuated immunity against $B$. cinerea.}

To explore the involvement of SISAPs in tomato immunity against $B$. cinerea, we performed functional analyses of each of the SISAP genes through a VIGS approach. For this purpose, fragments of 321 to $378 \mathrm{bp}$ for each of the SISAP genes were amplified and were cloned into the VIGS vector pTRV (Liu et al. 2002). Standard VIGS procedure with each of the pTRV-SISAP constructs and with negative (pTRV-GUS [ $\beta$-glucuronidase]) and positive (pTRV-SIPDS) controls was applied on 10-day-old seedlings. Under our experiment conditions, $>90 \%$ of the pTRV-SIPDS-infiltrated plants showed a bleaching phenotype at 2 weeks after agroinfiltration. The silencing efficiency for each of the SISAP genes in the corresponding pTRV-SISAP-infiltrated plants varied with a range of 55 to $80 \%$ (Supplementary Fig. S1), when the transcript level of a specific SISAP gene in the corresponding pTRV-SISAP-infiltrated plants was compared with that in the pTRV-GUS-infiltrated plants. Silencing specificity was also examined with SISAP4 as an example, because SISAP4 was the focus in this study. The transcript level of SISAP4 was significantly decreased in the pTRV-SISAP4-infiltrated plants, estimated to be approximately $20 \%$ of that in the pTRV-GUSinfiltrated plants, whereas the transcript levels of the other SISAP genes in the pTRV-SISAP4-infiltrated plants were comparable to those in the pTRV-GUS-infiltrated plants. Overall, the silencing efficiency and specificity of the target $S I S A P$ genes were satisfied for further experiments. During our repeated experiments, we found that the pTRV-SISAP10-infiltrated plants exhibited an obvious accelerated yellowing phenotype and contained lower content of chlorophyll, as compared with those of the pTRVGUS-infiltrated plants (Supplementary Fig. S2). No obvious 
change or defect in growth and development was observed, in tomato plants, with silencing of each of the other 12 SISAP genes.

We then examined the $B$. cinerea-caused disease phenotype on plants with silencing of each of the SISAP genes by comparing the $B$. cinerea-caused disease phenotype between the single SISAP-silenced plants and unsilenced plants using both detached-leaf inoculation and whole-plant inoculation assays. In detached-leaf inoculation assays, the B. cinereacaused disease lesions on leaves of the pTRV-SISAP4- and pTRV-SISAP10-infiltrated plants were significantly larger (Fig. 2A), leading to an approximately $40 \%$ of increase in size (Fig. 2C), as compared with those on leaves of the pTRVGUS-infiltrated plants, at 3 days postinoculation (dpi). In whole-plant inoculation assays, the $B$. cinerea-caused disease on the pTRV-SISAP4- or pTRV-SISAP10-infiltrated plants was much severe, resulting in the death and decay of approximately $90 \%$ of the infected plants, as compared with the pTRV-GUS-infiltrated plants, at 6 dpi (Fig. 2B). Quantification of in-planta fungal growth by quantitative reverse transcription-polymerase chain reaction (qRT-PCR) analysis of the transcript level of the $B$. cinerea BcActin gene showed that the fungal growth, as judged by the fold increase of BcActin/SlActin, in the pTRV-S1SAP4-infiltrated plants was significantly higher than those in the pTRV-GUS-infiltrated plants, leading to 1.6- and 2.1-fold increase at 24 and $48 \mathrm{hpi}$, respectively (Fig. 2D). By contrast, the $B$. cinerea-caused disease on plants with silencing of each of the remaining SISAP genes was indistinguishable from those on the pTRVGUS-infiltrated plants in both the detached-leaf inoculation and whole-plant inoculation assays (Fig. 2A, B and C). These data indicate that SISAP4 and SISAP10 play roles in immunity against $B$. cinerea. Due to the accelerated yellowing phenotype in the pTRV-SISAP10-infiltrated plants, we focused on SISAP4 to explore its mechanism in tomato immunity against B. cinerea.
Transient expression of SlSAP4 in N. benthamiana resulted in increased immunity against $B$. cinerea.

To confirm the function of SISAP4 in immunity, we examined whether transient expression of SlSAP4 in $N$. benthamiana could affect resistance to $B$. cinerea. To this end, an empty green fluorescent protein (GFP) vector and a SISAP4-GFP fusion construct were separately agroinfiltrated into leaves of $N$. benthamiana plants, and B. cinerea-caused disease phenotype was determined. In SISAP4-GFPinfiltrated in leaves, the transcript level of SISAP4 increased gradually over time during a period of $48 \mathrm{~h}$ (Fig. 3A) and the SISAP4-GFP fusion protein was also detected (Fig. 3B). In detached-leaf inoculation assays, the $B$. cinerea-caused lesions on leaves of the GFP-infiltrated plants, buffer-infiltrated plants, and untreated mock plants were comparable; however, the disease lesions on leaves of the SISAP4-GFP-infiltrated plants were significantly smaller than those in leaves of the GFPinfiltrated, buffer-infiltrated, and untreated mock plants (Fig. 3C), leading to an approximately $35 \%$ reduction in lesion size at 5 dpi (Fig. 3D). Similarly, the fungal growth in leaves of the GFP-infiltrated, buffer-infiltrated, and untreated mock plants was indistinguishable; however, the fungal growth in leaves of the SISAP4-GFP-infiltrated plants was markedly reduced by 40 and $31 \%$ at 24 and 48 hpi, respectively, as compared with those in leaves of GFP-infiltrated, bufferinfiltrated, and untreated mock plants (Fig. 3E). These data indicate that transient expression of SISAP4 in N. benthamiana plants provided an increased resistance to $B$. cinerea, further confirming a positive contribution of SlSAP4 to immunity against $B$. cinerea.

\section{Silencing of the SlSAP4 attenuated defense response against $B$. cinerea.}

To explore the molecular mechanism of SISAP4 in immunity against $B$. cinerea, we analyzed and compared the changes in accumulation of reactive oxygen species (ROS), contents of
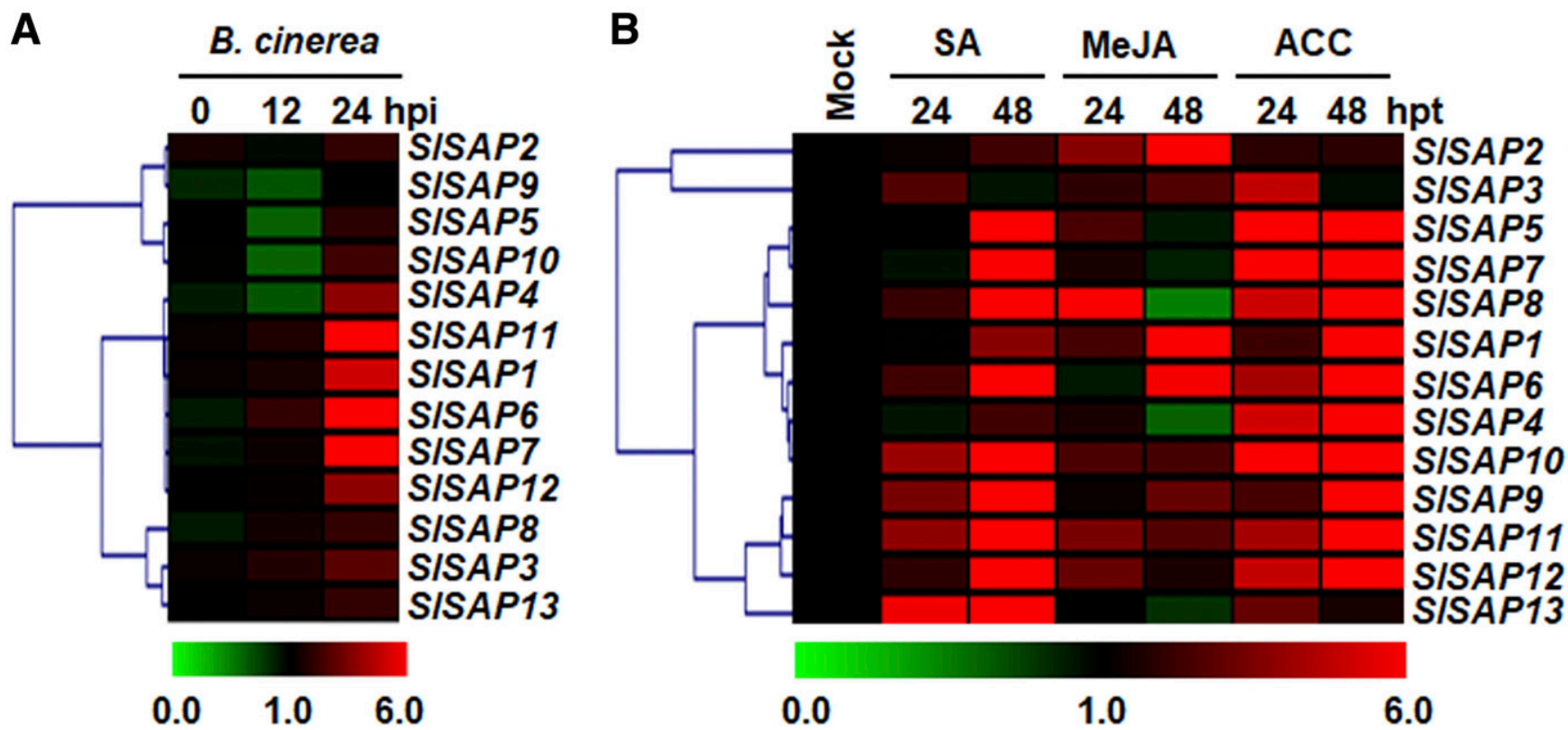

Fig. 1. Expression patterns of the SISAP genes in response to Botrytis cinerea and defense signaling hormones. A, Expression patterns of SISAP genes in response to $B$. cinerea. Four-week-old plants were inoculated by foliar spraying with spore suspension $\left(2 \times 10^{5}\right.$ spores per milliliter $)$ of $B$. cinerea or a similar volume of buffer, as a mock-inoculation control. B, Expression patterns of SlSAP genes after treatment with defense signaling hormones. Four-week-old tomato plants were treated by foliar spraying $1 \mathrm{mM}$ salicylic acid (SA), $100 \mu \mathrm{M}$ methyl jasmonate (MeJA), $100 \mu \mathrm{M} 1$-aminocyclopropane-1-carboxylicacid (ACC) or an equal volume of solution, as controls. Leaf samples were collected at indicated timepoints and the obtained quantitative reverse transcriptionpolymerase chain reaction data were normalized using the transcript level of SlActin. Relative expression of the genes of interest is presented in a heatmap, using fold-change values transformed to $\log _{2}$ format by MeV4.9. Genes were clustered according to their expression profiles. hpi $=$ hours postinoculation; hpt $=$ hours posttreatment. 
defense signaling hormones, and expression levels of defense genes between the pTRV-SISAP4-infiltrated and pTRV-GUSinfiltrated plants. Without infection of $B$. cinerea, no difference in accumulation of $\mathrm{H}_{2} \mathrm{O}_{2}$, as detected by 3, 3-diaminobenzidine
(DAB) staining, was observed in leaves of the pTRV-SISAP4and pTRV-GUS-infiltrated plants (Fig. 4A and B). By contrast, infection of $B$. cinerea induced significantly the accumulation of $\mathrm{H}_{2} \mathrm{O}_{2}$ in leaves of both the pTRV-SISAP4- and
A
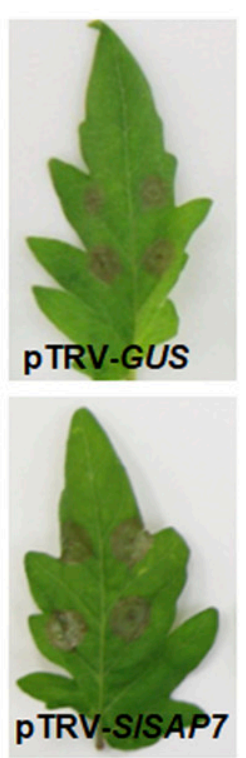

B
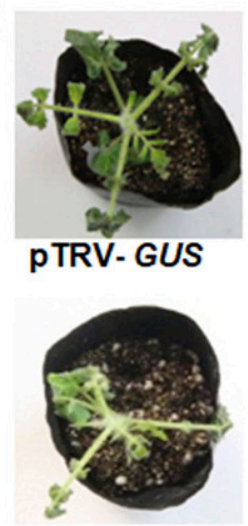
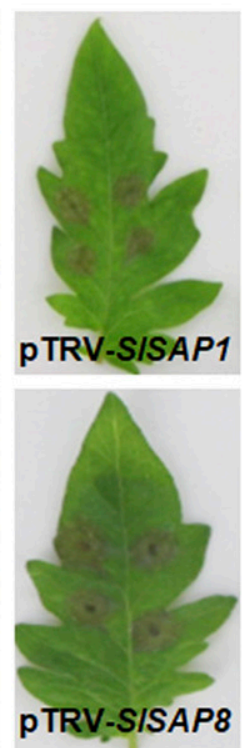
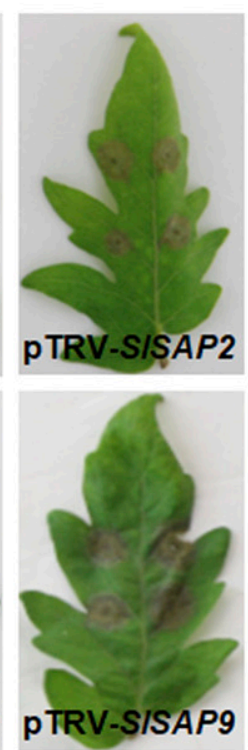
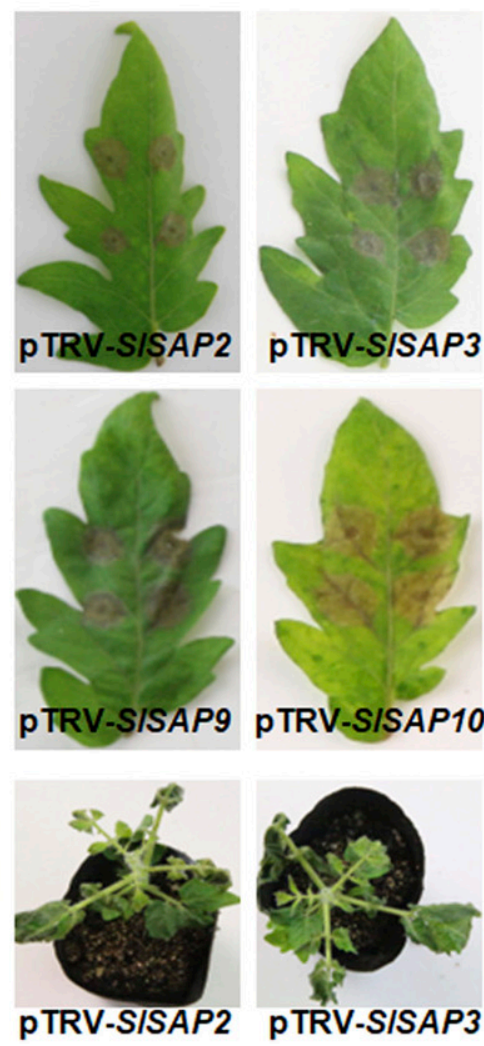

pTRV-SISAP1
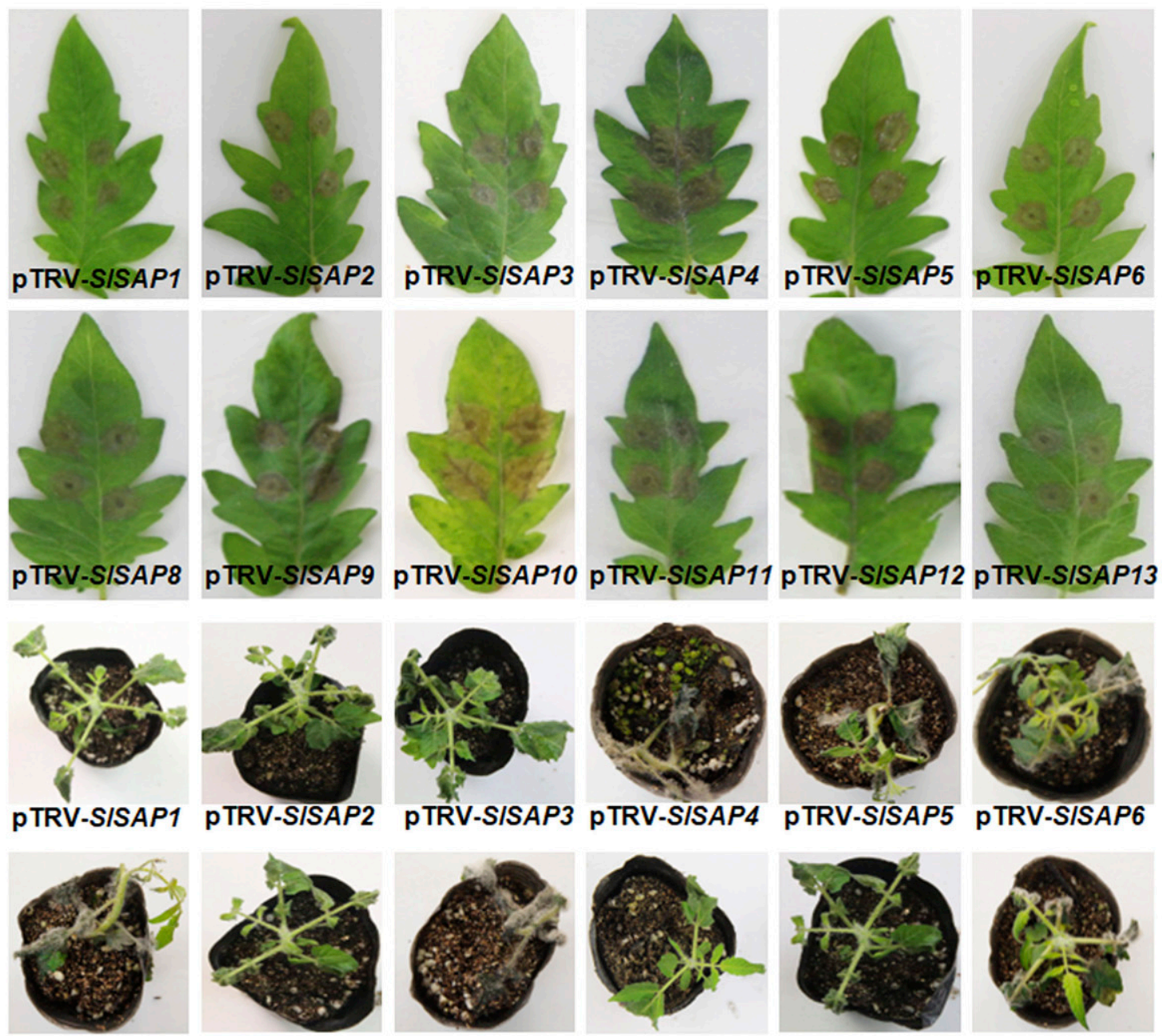

pTRV-SISAP7 PTRV-SISAP8

PTRV-SISAP9

C

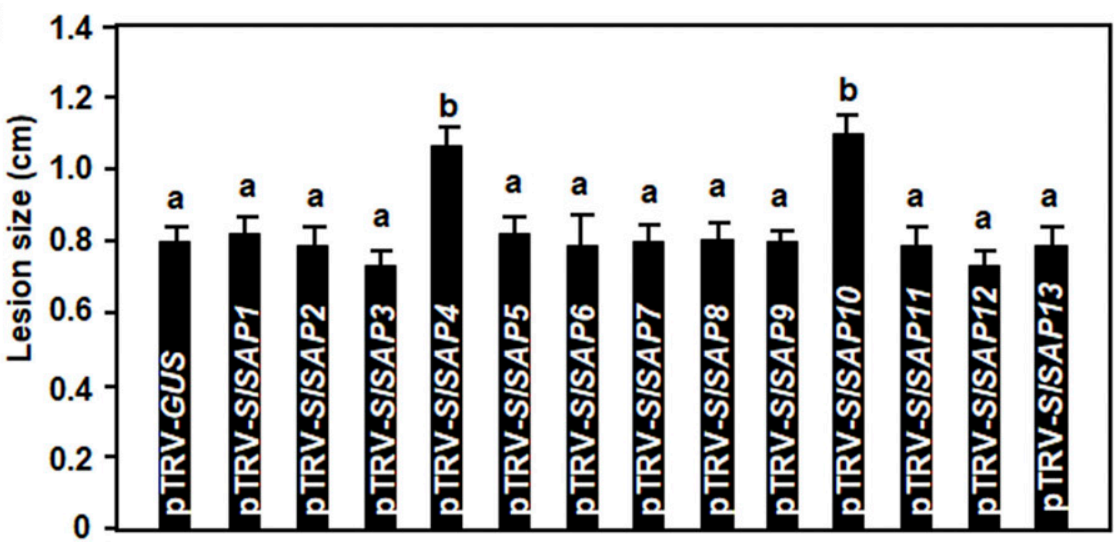

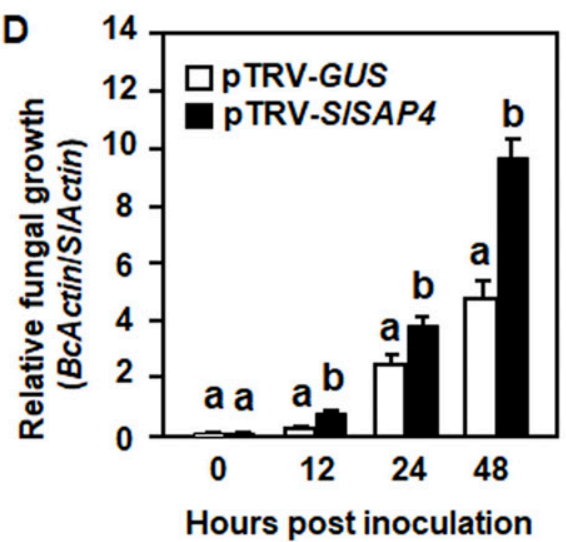

Fig. 2. Silencing of SISAP4 resulted in reduced resistance to Botrytis cinerea. Ten-day-old seedlings were infiltrated with agrobacteria carrying pTRV-SISAPs or pTRV-GUS, and disease assays were carried out at 4 weeks after agroinfiltration by dropping a spore suspension $\left(1 \times 10^{5}\right.$ spores per milliliter) of $B$. cinerea on detached leaves or foliar spraying with spore suspension $\left(2 \times 10^{5}\right.$ spores per milliliter) onto leaves of whole plants. A, Disease phenotype on leaves of the pTRV-SISAPs- or pTRV-GUS-infiltrated plants in detached-leaf inoculation assays. Photographs were taken at 3 days postinoculation (dpi). B, Disease phenotype of pTRV-SISAP- and pTRV-GUS-infiltrated plants after spraying with B. cinerea. C, Lesion sizes on leaves of the pTRV-SISAP- or pTRVGUS-infiltrated plants in detached-leaf inoculation assays. Lesion sizes were measured at 3 dpi on a minimum of 20 leaves in each experiment. D, Fungal growth in leaves of pTRV-SISAP4- and pTRV-GUS-infiltrated plants after inoculation with $B$. cinerea. Photographs were taken at 6 dpi. Fungal growth in planta was estimated at the indicated timepoints by analyzing the transcript levels of B. cinerea BcActin and tomato SlActin as an internal control and are shown as ratios of the transcript level of BcActin to that of SlActin. Data presented in C and D are the means \pm standard deviation from three independent experiments and different letters above the columns indicate significant difference at $P<0.05$. 

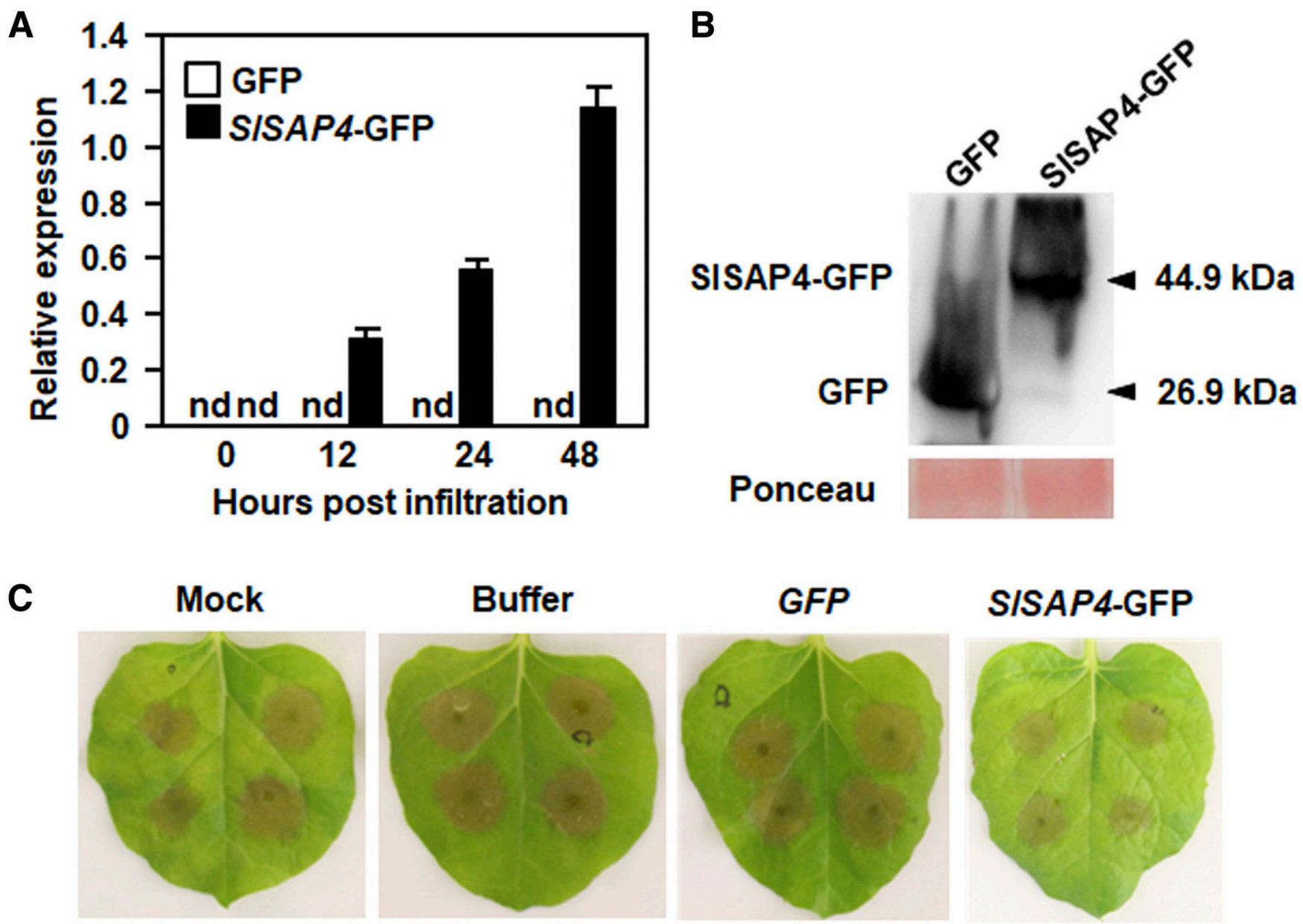

Buffer

GFP

SISAP4-GFP

D
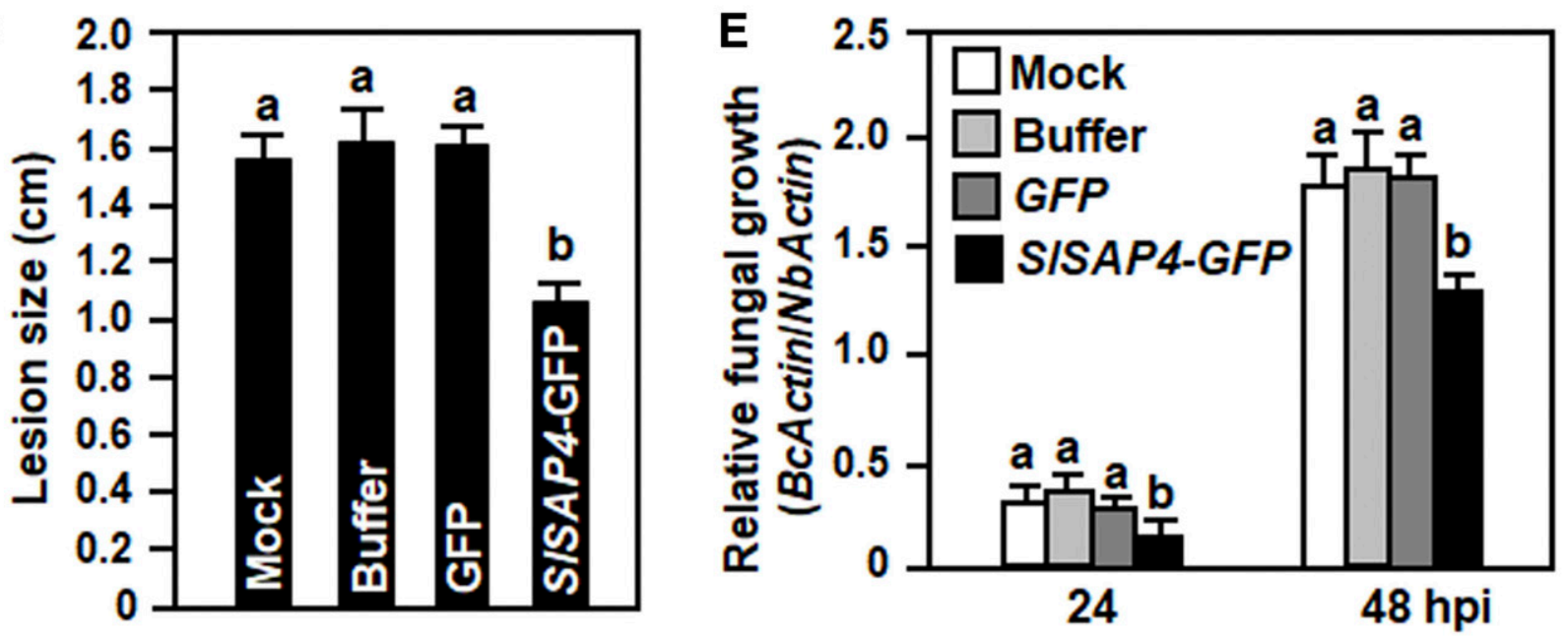

Fig. 3. Transient expression of SISAP4 in Nicotiana benthamiana led to an increased resistance to Botrytis cinerea. A, Transcript level of SlSAP4 in agroinfiltrated leaves. Agrobacteria carrying pFGC-SISAP4 or pFGC-eGFP were infiltrated into leaves of $N$. benthamiana and the transcript level of SISAP4 was analyzed by quantitative reverse transcription-polymerase chain reaction (qRT-PCR). Relative transcript levels were calculated by comparison with the corresponding values at $0 \mathrm{~h}$ (as a control) after infiltration. B, Immunoblot detection of SISAP4-GFP fusion protein in agroinfiltrated leaves at $48 \mathrm{~h}$ after agroinfiltration. A green fluorescent protein (GFP)-specific antibody was used for detection of GFP-fusion protein. Equal loading of total proteins was examined by Ponceau staining. $\mathbf{C}$ and $\mathbf{D}, B$. cinerea-caused disease symptoms and lesion size on agroinfiltrated leaves, buffer-infiltrated, and untreated mock leaves. Leaves infiltrated with agrobacteria carrying pFGC-SISAP4 or pFGC-eGFP or with buffer, as wounding controls, and untreated leaves without infiltration, as mock controls, were inoculated by dropping spore suspension $\left(2 \times 10^{5}\right.$ spores per milliliter) of $B$. cinerea, and lesion sizes were measured at 5 days postinoculation. E, Fungal growth of $B$. cinerea in agroinfiltrated leaves. Fungal growth in planta was estimated by qRT-PCR, analyzing the transcript levels of BcActin and NbActin, as an internal control, at the indicated timepoints after inoculation. Data presented in A, B and D are the means \pm standard deviation from three independent experiments and different letters above the columns indicate significant difference at $P<0.05$. 
A

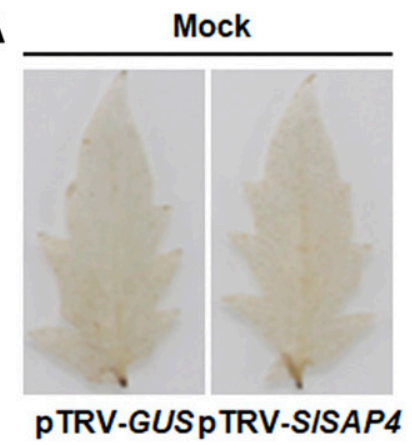

B. cinerea

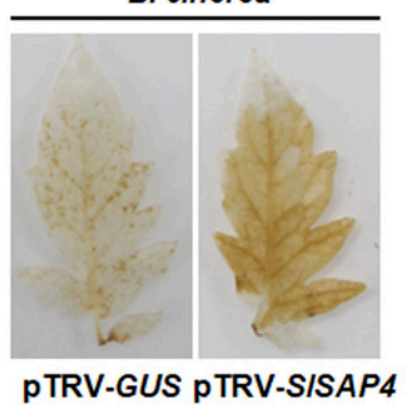

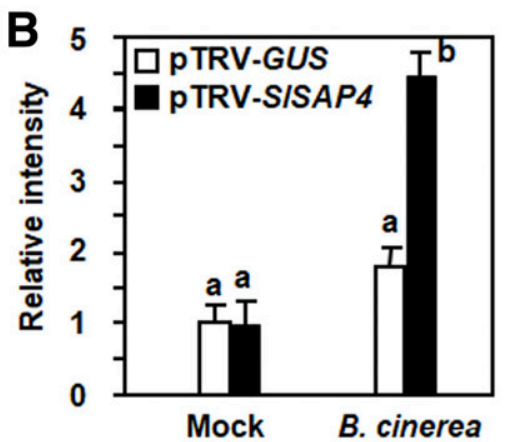
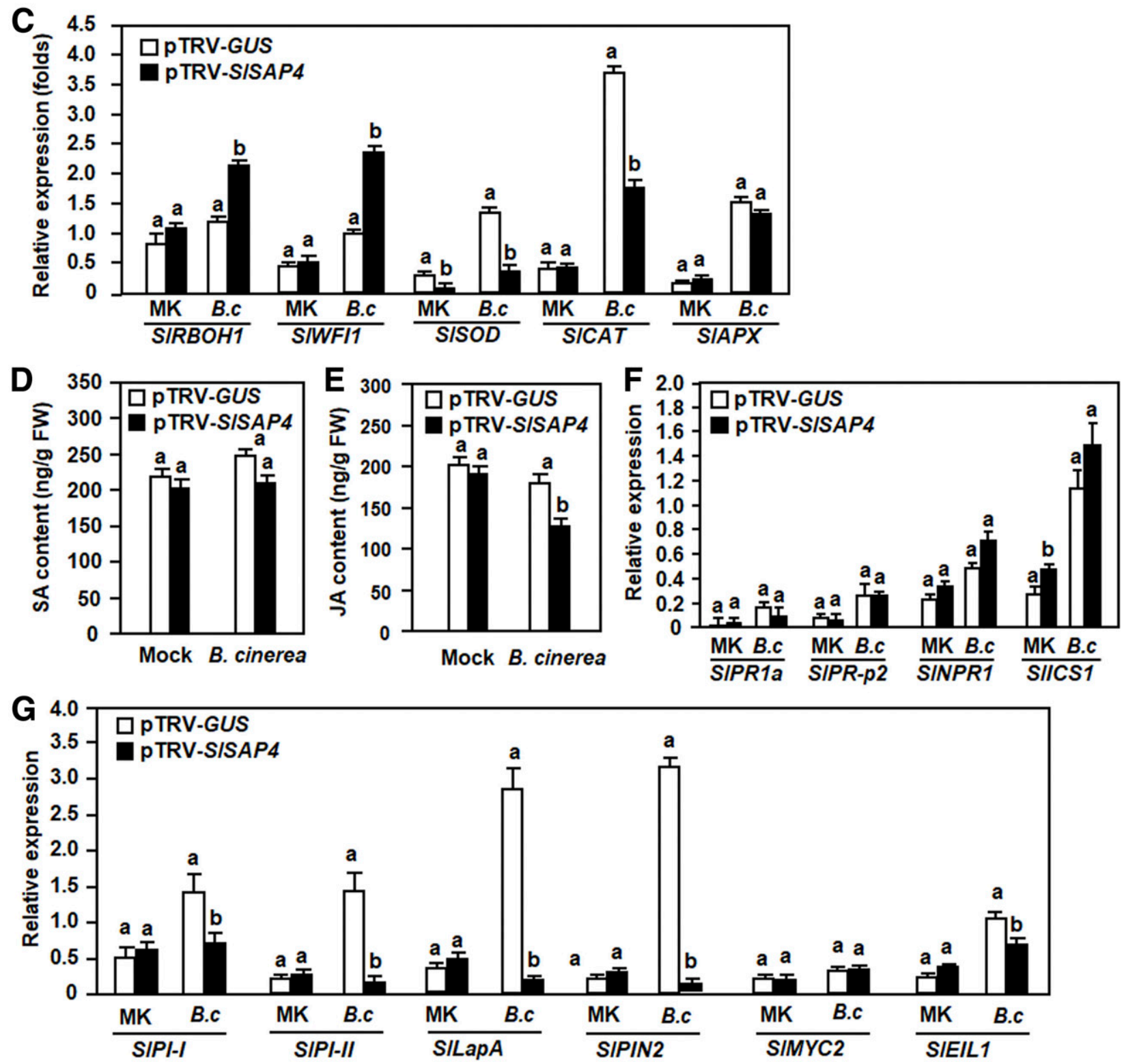

Fig. 4. Silencing of SISAP4 increased accumulation of $\mathrm{H}_{2} \mathrm{O}_{2}$ and affected the expression of defense genes after infection of Botrytis cinerea. Ten-day-old seedlings were infiltrated with agrobacteria carrying pTRV-SISAP4 or pTRV-GUS and the virus-induced gene silencing-infiltrated plants were inoculated with spore suspension of $B$. cinerea or with buffer solution as a mock inoculation control at 4 weeks after agroinfiltration. Leaves from six individual plants were collected at $24 \mathrm{~h}$ postinoculation (hpi). A, Accumulation of $\mathrm{H}_{2} \mathrm{O}_{2}$ by 3, 3-diaminobenzidine (DAB) staining and $\mathbf{B}$, relative intensity of DAB-stained $\mathrm{H}_{2} \mathrm{O}_{2}$. C, Expression of reactive oxygen species generating- and scavenging-related genes in pTRV-SISAP4- and pTRV-GUS-infiltrated plants with or without infection of B. cinerea at 24 hpi. D, Salicylic acid (SA) and E, jasmonate (JA) contents in pTRV-SISAP4- and pTRV-GUS-infiltrated plants with or without infection of B. cinerea at 24 hpi. F, Expression of SA-mediated signaling regulatory and responsive defense genes and $\mathbf{G}$, expression of JA and ethylene-mediated signaling regulatory and responsive defense genes. Data presented in $\mathrm{B}$ to $\mathrm{G}$ are the means \pm standard deviation from three independent experiments and different letters above the columns indicate significant difference at $P<0.05$. MK $=$ mock-inoculated control; B.c $=$ B. cinerea-inoculated treatment. 
pTRV-GUS-infiltrated plants; however, the $B$. cinerea-infected leaves of the pTRV-SISAP4-infiltrated plants accumulated much more $\mathrm{H}_{2} \mathrm{O}_{2}$ (Fig. 4A), leading to a 2.5 -fold increase in relative $\mathrm{DAB}$ staining intensity, as compared with those in the pTRV-GUS-infiltrated plants, at 24 hpi (Fig. 4B). The expression changes of some selected ROS generating- and scavenging-related genes matched the changes in $\mathrm{H}_{2} \mathrm{O}_{2}$ accumulation in the pTRV-SISAP4- and pTRV-GUS-infiltrated plants with or without infection of $B$. cinerea (Fig. 4C). The expression levels of two RBOH genes, SIRBOH1 and SlWFIl, were comparable in the pTRV-SISAP4- and pTRV-GUSinfiltrated plants without $B$. cinerea infection but increased significantly in the pTRV-S1SAP4-infiltrated plants after infection of $B$. cinerea, leading to 0.9- and 1.4-fold increases, as compared with those in the pTRV-GUS-infiltrated plants (Fig. $4 C)$. The expression level of SISOD in the pTRV-SISAP4infiltrated plants was decreased markedly, leading to 3.1- and 3.9-fold reductions, as compared with those in the pTRVGUS-infiltrated plants with or without $B$. cinerea infection (Fig. 4C). Although the expression level of SlCAT in the pTRVSISAP4- and pTRV-GUS-infiltrated plants without $B$. cinerea infection was comparable, the level in the pTRV-SISAP4infiltrated plants after $B$. cinerea infection was decreased significantly, resulting in 1.2-fold reduction, as compared with that in the pTRV-GUS-infiltrated plants (Fig. 4C). The expression levels of SIAPX were comparable in the pTRV-SISAP4- and pTRV-GUS-infiltrated plants with or without infection of $B$. cinerea (Fig. 4C). These data indicate the silencing of SISAP4 disturbed the balance between ROS generation and scavenging and thus led to $\mathrm{H}_{2} \mathrm{O}_{2}$ accumulation upon $B$. cinerea infection.

The SA content in leaves of the pTRV-SISAP4- and pTRVGUS-infiltrated plants without infection of $B$. cinerea was comparable and remained unchanged after infection of B. cinerea (Fig. 4D). Although the JA content in leaves of the pTRV-SISAP4- and pTRV-GUS-infiltrated plants without infection of B. cinerea were comparable, the JA content in leaves of the pTRV-SISAP4-infiltrated plants decreased, leading to $30 \%$ of reduction, as compared with those in leaves of the pTRV-GUS-infiltrated plants at 24 hpi with $B$. cinerea (Fig. 4E). In mock-inoculated pTRV-SISAP4- and pTRV-GUSinfiltrated plants, the expression levels of some selected regulatory genes of the JA/ET and SA signaling pathways and their corresponding defense genes were comparable, except for an increase of expression of SIICS1 in pTRV-SISAP4-infiltrated plants (Fig. 4F and G). No significant difference in the expression levels of the SA signaling regulatory genes SINPRI and SIICSI and the corresponding defense genes SIPRIa and SIPR-p2 was observed in the pTRV-SISAP4- and pTRVGUS-infiltrated plants at 24 hpi by $B$. cinerea (Fig. 4F). By contrast, the expression of the JA/ET signaling regulatory gene SIEILI and the corresponding defense genes SIPI-I, SIP-II, SlLapA, and SIPIN2 in the pTRV-SISAP4- and pTRV-GUSinfiltrated plants increased markedly at 24 hpi with $B$. cinerea; however, the expression levels of these regulatory and defense genes in the pTRV-SISAP4-infiltrated plants decreased significantly, showing 1.6-, 2.1-, 9.9-, 15.3-, and 32.7-fold reductions, respectively, as compared with those in the pTRVGUS-infiltrated plants (Fig. 4G). No difference in the expression levels of SIMYC2 was observed in the pTRV-SISAP4- and pTRV-GUS-infiltrated plants after inoculation with $B$. cinerea (Fig. 4G). These data indicate that silencing of SISAP4 attenuated the JA/ET signaling and defense response upon infection of $B$. cinerea.

To further confirm the involvement of JA signaling in the SISAP4-mediated immunity against $B$. cinerea, we examined whether exogenous application of MeJA could rescue the resistance of the pTRV-S1SAP4-infitrated plants against $B$. cinerea. In detached-leaf inoculation and whole-plant inoculation assays, the $B$. cinerea-caused disease severity on the MeJA-treated pTRV-SISAP4-infiltrated plants was less severe than that in the MeJA-untreated pTRV-SISAP4-infiltrated plants (Fig. 5A and C). Similarly, the lesion sizes at 3 dpi on detached leaves and fungal growth at 24 and 48 hpi in leaves of the MeJA-treated pTRV-SISAP4-infiltrated plants were significantly reduced, when compared with those in leaves of the MeJA-untreated pTRV-SISAP4-infiltrated plants (Fig. 5B and D). Furthermore, $B$. cinerea-induced $\mathrm{H}_{2} \mathrm{O}_{2}$ accumulation in leaves of the MeJA-treated pTRV-SISAP4-infiltrated plants was also reduced in comparison with that in leaves of the MeJA-untreated pTRV-SISAP4-infiltrated plants (Fig. 5E and F). These data indicate that exogenous application of MeJA partially restored the resistance of the SISAP4-silenced plants against $B$. cinerea, further confirming the involvement of JA signaling in SISAP4-mediated immunity against $B$. cinerea.

\section{SISAP4 did not have ubiquitin E3 ligase activity in vitro.}

It has been shown that Arabidopsis AtSAP5 and AtSAP9 and rice OsSAP7 possess ubiquitin E3 ligase activity in vitro (Kang et al. 2011, 2017; Sharma et al. 2015). To examine whether SISAP4 had ubiquitin E3 ligase activity, we expressed a His-SISAP4 fusion in Escherichia coli cells and purified the recombinant His-SISAP4 protein. In the standard ubiquitination reactions containing the components Ub, E1, and E2, the Arabidopsis AtPUB13 (Liao et al. 2017), as a positive control, catalyzed the formation of multiubiquitin conjugates, whereas a collection from E. coli expressing an empty His vector did not form any multiubiquitin conjugate (Fig. 6A), indicating that our ubiquitination assays worked properly. However, no multiubiquitin conjugates were observed in reactions containing standard components of $\mathrm{Ub}$, E1, E2, and His-S1SAP4 or in reactions lacking E1, E2, or HisSISAP4 (Fig. 6A). Therefore, it seems that SISAP4 did not have ubiquitin E3 ligase activity in vitro.

\section{SISAP4 interacted with SIRAD23d.}

Because SISAP4 plays an important role in immunity against $B$. cinerea, we tried to identify its interacting partners through yeast two-hybrid $(\mathrm{Y} 2 \mathrm{H})$ screening to elucidate the possible mechanism of SISAP4 in immunity. To this end, a Y2H library was generated using cDNAs synthesized with total RNA from B. cinerea-infected tomato leaves and screened using SISAP4 as bait. Approximately $10^{6}$ yeast cells were screened in two independent screening assays and a total of 58 putative positive clones were initially obtained. After sequencing of the rescued plasmids, 44 positive clones contained in-frame sequences encoding 10 different proteins, while the remaining 14 clones contained shifted-frame sequences (Supplementary Fig. S3). We further examined and validated by $\mathrm{Y} 2 \mathrm{H}$ the interaction between SISAP4 and each of these putative interacting partners and identified three of them, Solyc08g083120 (ubiquitinribosomal fusion protein), Solyc01g056940 (ubiquitin), and Solyc02g063130 (RAD23 UV excision repair protein), as S1SAP4 interacting partners. As the Arabidopsis AtRAD23d has been shown to interact with AtSAP9 (Kang et al. 2017), we thus focused on the interaction of SIRAD23d (nomenclature of the tomato SIRAD23 proteins is provided below) with SISAP4 and its functions in immunity against $B$. cinerea. Biomolecular florescence complementation (BiFC) and coimmunoprecipitation (co-IP) assays were performed to further confirm the SISAP4-SIRAD23d interaction in planta. In BiFC assays, combined mixtures of agrobacteria harboring the $\mathrm{N}$-terminal half of yellow fluorescent protein (nYFP) or the C-terminal half (cYFP), SISAP4-nYFP or cYFP, nYFP or SIRAD23d-cYFP, and SISAP4-nYFP or SIRAD23d-cYFP, were separately 
A

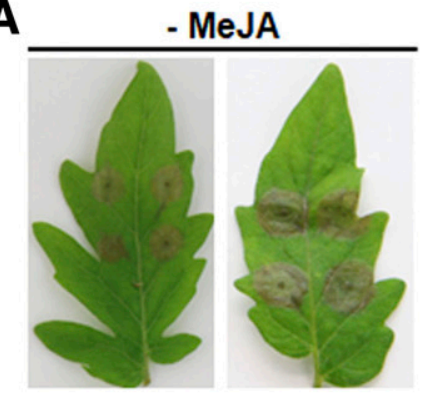

pTRV-GUS pTRV-SISAP4
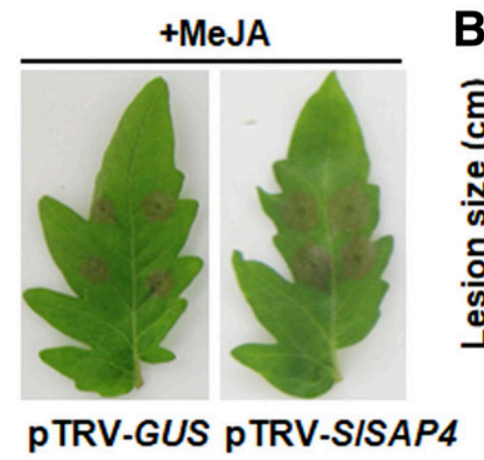
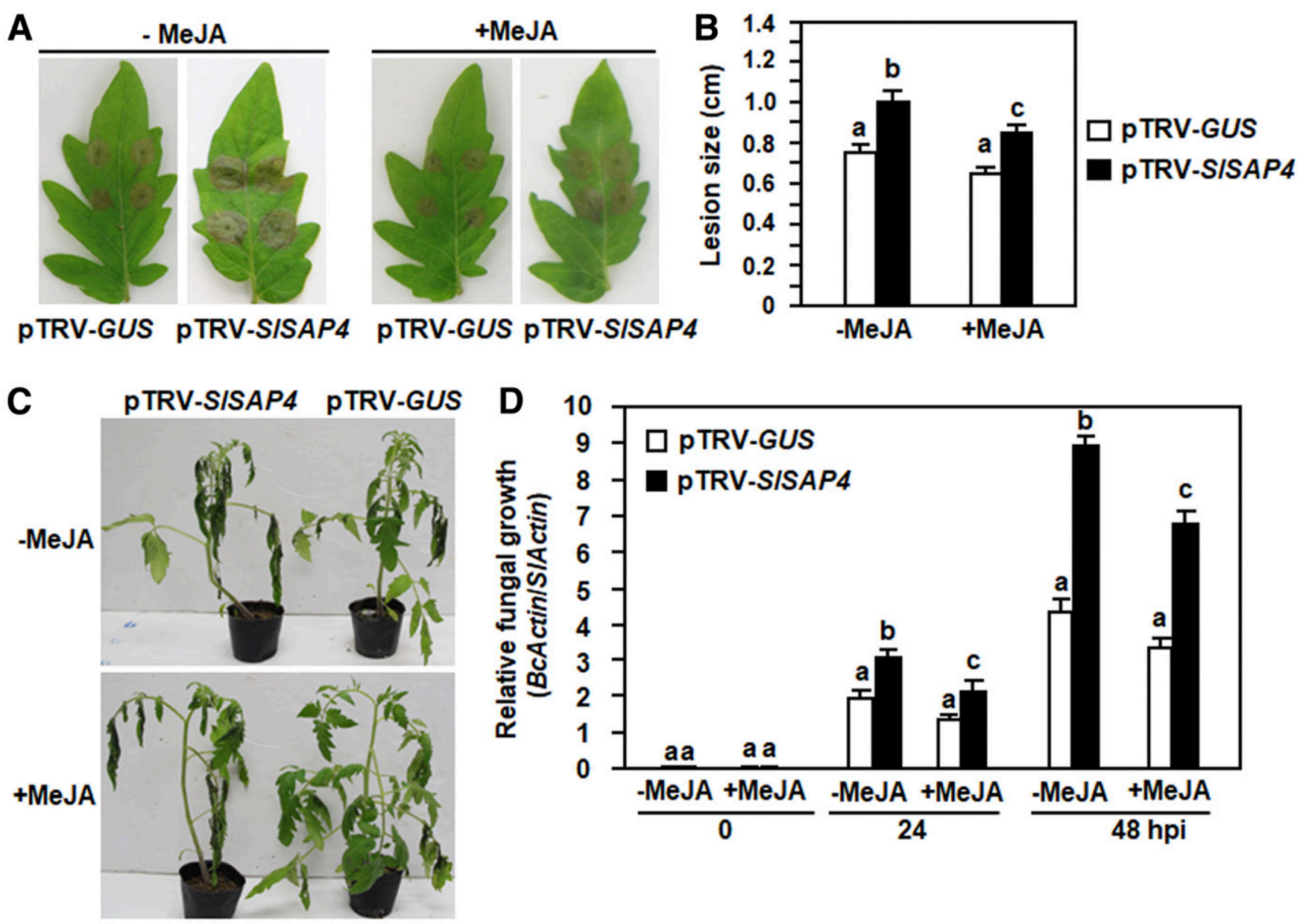

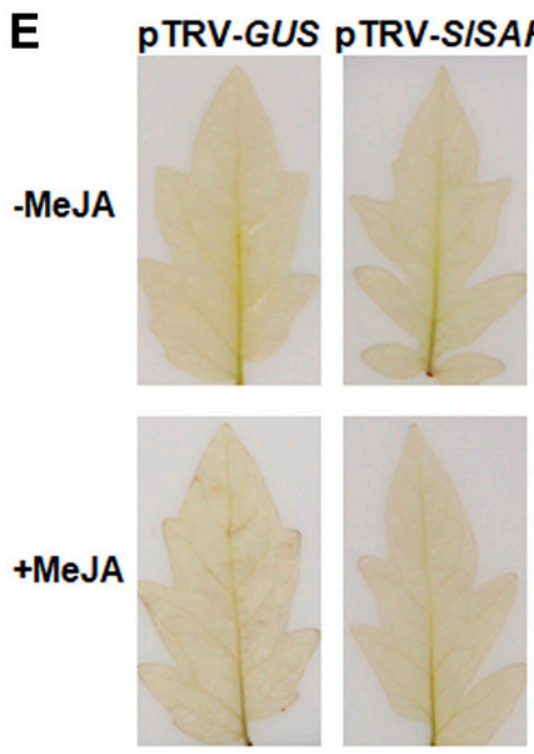

Mock
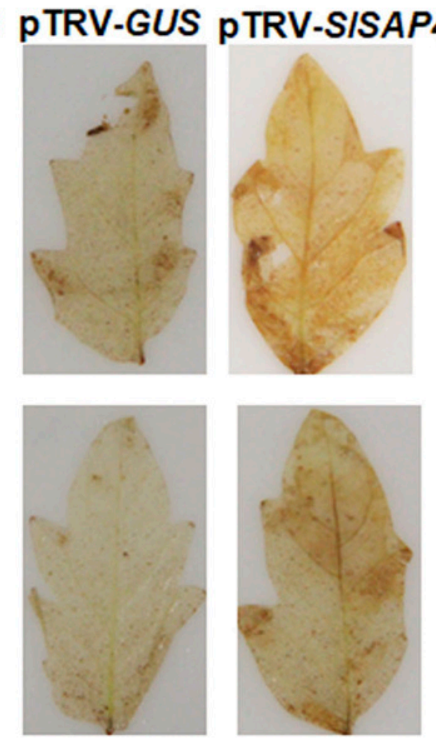

B. cinerea

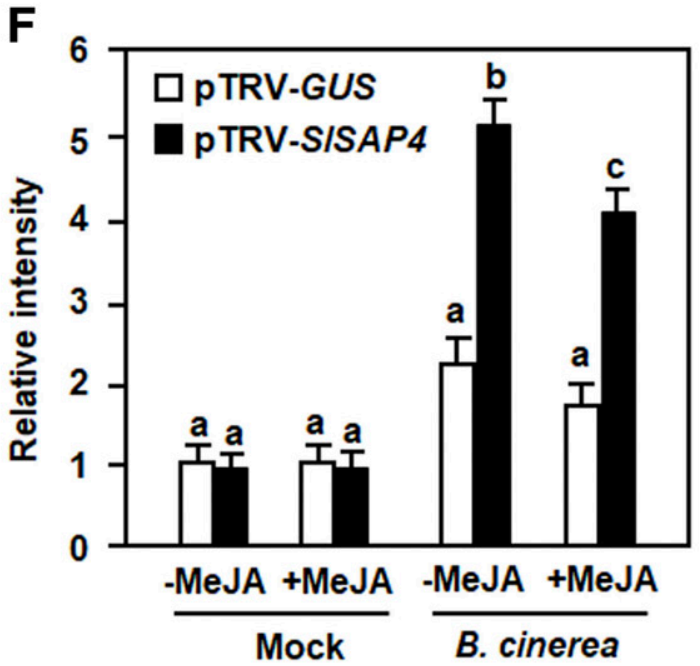

Fig. 5. Exogenous application of methyl jasmonate (MeJA) partially restored the resistance of the SlSAP4-silenced plants against Botrytis cinerea. Ten-day-old seedlings were infiltrated with agrobacteria carrying pTRV-SISAP4 or pTRV-GUS and were treated by foliar spraying $100 \mu \mathrm{M}$ MeJA or an equal volume of solution, as controls, at 4 weeks after agroinfiltration. Disease assays were carried out, at $24 \mathrm{~h}$ after MeJA treatment, by dropping spore suspension $\left(1 \times 10^{5}\right.$ spores per milliliter) of $B$. cinerea on detached leaves or foliar spraying with spore suspension $\left(2 \times 10^{5}\right.$ spores per milliliter) onto leaves of whole plants. A, Disease phenotype and B, lesion size on inoculated detached leaves of the pTRV-SISAP4- and pTRV-GUS-infiltrated plants with or without MeJA treatment. Photographs were taken at 3 days postinoculation (dpi). C, Disease phenotype and D, fungal growth in leaves of $B$. cinerea-inoculated pTRV-SISAP4- and pTRV-GUS-infiltrated plants with or without MeJA treatment. Photographs were taken at 3 dpi. Fungal growth in planta was estimated at the indicated timepoints by analyzing the transcript levels of $B$. cinerea BcActin and tomato SlActin as an internal control, shown as ratios of the transcript level of BcActin to that of SlActin. E, Accumulation of $\mathrm{H}_{2} \mathrm{O}_{2}$ by 3, 3-diaminobenzidine (DAB) staining and $\mathbf{F}$, relative intensity of DAB-stained $\mathrm{H}_{2} \mathrm{O}_{2}$ in leaves of $B$. cinereainoculated pTRV-SISAP4- and pTRV-GUS-infiltrated plants with or without MeJA treatment. Data presented in B, D, and F are the means \pm standard deviation from three independent experiments and different letters above the columns indicate significant difference at $P<0.05$. 

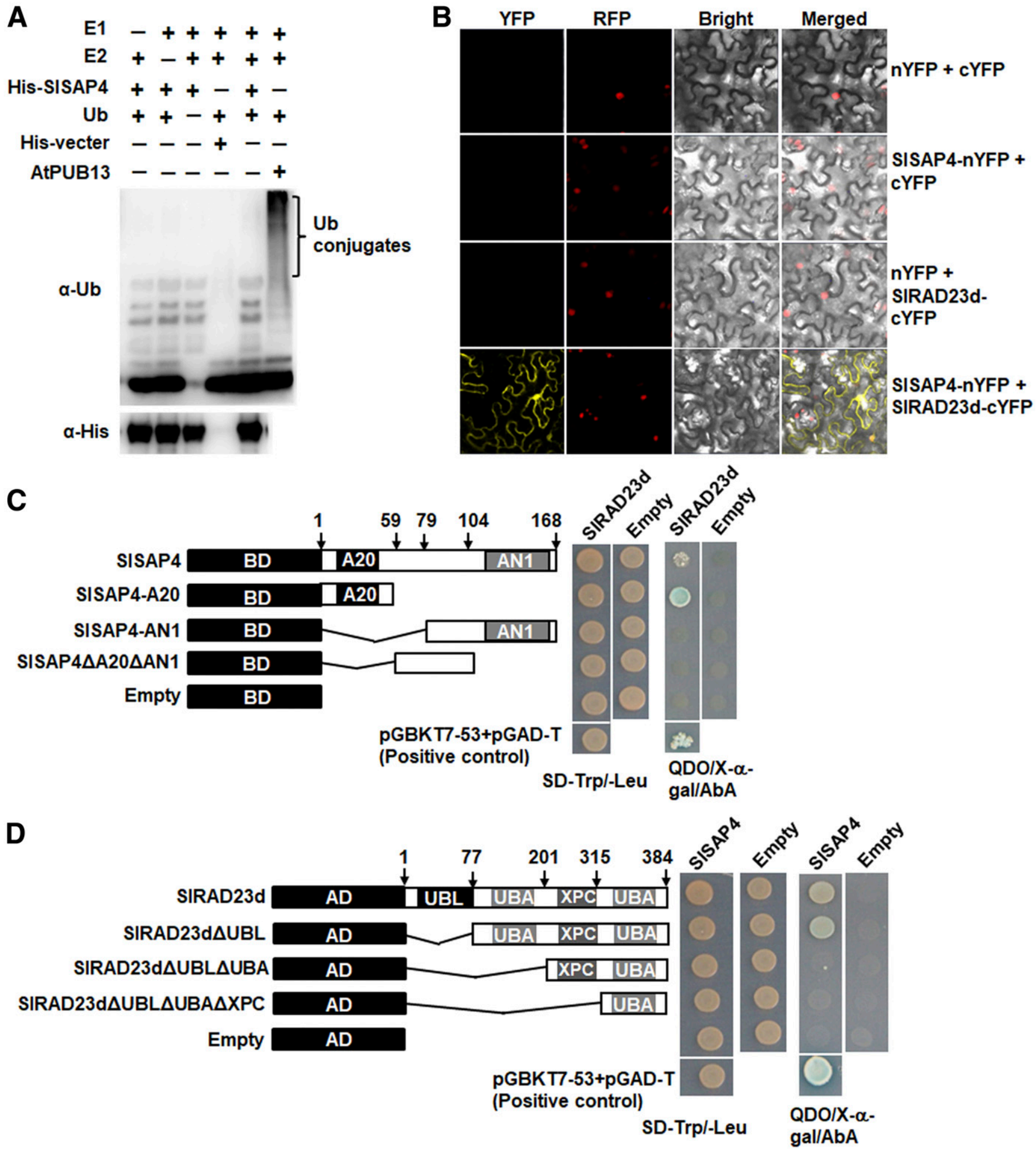

Fig. 6. SISAP4 did not possess ubiquitin E3 ligase activity but interacted with SIRAD23d. A, Detection of ubiquitin E3 ligase activity. An in-vitro ubiquitination assay was performed by incubating purified recombinant His-SISAP4 with or without human E1, E2 (UbcH2), and ubiquitin at $30^{\circ} \mathrm{C}$ for $3 \mathrm{~h}$. Ubiquitin conjugates were resolved by sodium dodecyl sulfate-polyacrylamide gel electrophoresis and were detected by immunoblotting using antiubiquitin antibody. A collection purified from bacteria expressing an empty His vector was used as a negative control, while the Arabidopsis AtPUB13 was used as a positive control. B, Biomolecular florescence complementation analysis of the interaction between SISAP4 and SIRAD23d. Combined mixtures of agrobacteria harboring the N-terminal half of yellow fluorescent protein (nYFP) or the the C-terminal half (cYFP), SISAP4-nYFP or cYFP, nYFP or SIRAD23dcYFP, and SISAP4-nYFP or SIRAD23d-cYFP were separately infiltrated into leaves of Nicotiana benthamiana and fluorescence was observed under a confocal microscope at $48 \mathrm{~h}$ after agroinfiltration. C and D, Determination of domains responsible for the interaction activity in SISAP4 and SIRAD23d proteins. Different deletion mutants of S1SAP4 were generated and were fused to the DNA-binding domain in pGBKT7 vector (C, left), while different deletion mutants of SIRAD23d were created and were fused to the DNA-activating domain in pGADT7 vector (D, left). Yeast cells cotransformed with indicated pairs of vectors were incubated on SD/-Trp/-Leu and SD/-Ade/-His/-Leu/-Trp + X- $\alpha$-gal/AbA (QDO/X- $\alpha$-gal/AbA) plates to test their interaction activity. Yeast cells cotransformed with pGBKT7 and pGADT7 empty vectors or with pGBKT7-53 and pGADT7-T were used as negative and positive controls, respectively. Arrows indicate the amino acid positions in SISAP4 (C) and SIRAD23d (D). The experiments were repeated three times with similar results. 
infiltrated into leaves of $N$. benthamiana plants expressing a known nucleus marker protein red fluorescent protein (RFP)H2B (Chakrabarty et al. 2007) and fluorescence was examined at $48 \mathrm{~h}$ after agroinfiltration. YFP fluorescence was not detected in leaves coinfiltrated with agrobacteria harboring nYFP or cYFP, SISAP4-nYFP or cYFP, nYFP or SIRAD23d-cYFP, and nYFP or SIRAD23d-cYFP, whereas significant YFP fluorescence was clearly observed in leaves coinfiltrated with agrobacteria harboring SISAP4-nYFP or SIRAD23d-cYFP (Fig. $6 \mathrm{~B})$. Notably, the fluorescence generated from the interaction of SISAP4 and SIRAD23d was observed in both nuclear and cytoplasmic compartments (Fig. 6B). Similarly, when transiently expressed in leaves of $N$. benthamiana plants, the GFPSISAP4 and GFP-SIRAD23d were localized in both nuclear and cytoplasmic compartments. In co-IP assays, mixtures of agrobacteria harboring SISAP4-HA (hemagglutinin) and GFPSIRAD23d or SISAP4-HA and GFP were infiltrated into leaves of $N$. benthamiana plants, and total proteins were extracted two days later and were immunoprecipitated with anti-GFP magnetic beads. Western blot using anti-HA and anti-GFP antibodies showed that the GFP-SIRAD23d fusion protein coimmunoprecipitated with SISAP4-HA while the GFP did not (Supplementary Fig. S4). Taken together, these data clearly demonstrate that SISAP4 and SIRAD23d interacted in planta and their interaction occurred in nuclear and cytoplasmic compartments of the cells.

To determine the domains or regions in SISAP4 and SIRAD23d that are responsible for the SISAP4-SIRAD23d interaction, a series of deletion mutants of the SISAP4 and SIRAD23d proteins were constructed and examined for their interaction activity (Fig. 6C and D). In Y2H assays, like the full SISAP4 protein, S1SAP4-A20, lacking the greater part of the linker region and the AN1 domain, interacted with SIRAD23d, whereas S1SAP4-AN1, lacking the A20 domain and partial linker region, and SISAP $4 \triangle \mathrm{A} 20 \Delta \mathrm{AN} 1$, the full linker region lacking the $\mathrm{A} 20$ and $\mathrm{AN} 1$ domains, did not interact with SIRAD23d (Fig. 6C). Similarly, like the full SIRAD23d protein, SIRAD23d $\triangle$ UBL lacking the N-terminal UBL domain interacted with SISAP4, whereas SIRAD23d $\triangle \mathrm{UBL} \Delta \mathrm{UBA}$ with deletion of the N-terminal UBL and the first UBA domains and SIRAD23d $\triangle \mathrm{UBL} \triangle \mathrm{UBA} \triangle \mathrm{XPC}$ with deletion of the $\mathrm{N}$-terminal UBL, the first UBA, and the XPC/RAD4 domains lost the interaction activity with SISAP4 (Fig. 6D). These results indicate that the A20 domain in S1SAP4 and the UBA domains in SIRAD23d are critical for the SISAP4-SIRAD23d interaction.

\section{SIRAD23d positively contributed to immunity against $B$. cinerea.}

Having proven the interaction between SISAP4 and SIRAD23d, we then reasoned whether SIRAD23d played a role in immunity against $B$. cinerea. For this end, we examined the responsiveness of SIRAD23d to $B$. cinerea. As shown in Figure 7A, the expression level of SIRAD23d in mockinoculated plants remained unchanged during a period of $48 \mathrm{~h}$, whereas the expression level in B. cinerea-inoculated plants increased significantly, leading to an approximately fivefold increase over that in mock-inoculated plants at 48 hpi. To explore the involvement of $S I R A D 23 d$ in immunity against $B$. cinerea, we examined the $B$. cinerea-caused disease phenotype in the pTRV-SIRAD23d-infiltrated plants compared with that in the pTRV-GUS-infiltrated plants. The transcript level of SIRAD23d in the pTRV-SIRAD23d-infiltrated plants was approximately $30 \%$ that in the pTRV-GUS-infiltrated plants and silencing of SIRAD23d did not affect the transcript levels of other SIRAD23 genes. In detached-leaf inoculation assays, the $B$. cinerea-caused disease lesions on leaves of the
pTRV-SIRAD23d-infiltrated plants were larger (Fig. 7B) and showed an average of $36 \%$ increase over those on leaves of the pTRV-GUS-infiltrated plants (Fig. 7C). In whole-plant inoculation assays, the $B$. cinerea-caused disease symptoms in the pTRV-SIRAD23d-infiltrated plants were much more severe and most of the plants died and decayed at $6 \mathrm{dpi}$, as compared with those in the pTRV-GUS-infiltrated plants (Fig. 7D). Quantification of in-planta fungal growth showed that the fungal biomass in the pTRV-SIRAD23d-infiltrated plants was significantly higher than that in the pTRV-GUS-infiltrated plants, showing an approximately $40 \%$ increase at $24 \mathrm{hpi}$ (Fig. $7 \mathrm{E})$. These data suggest that silencing of SIRAD23d resulted in decreased resistance to $B$. cinerea, indicating that SIRAD23d, like its interacting partner SISAP4, plays a role in immunity against $B$. cinerea.

\section{Interaction of SISAP4 with other SIRAD23 proteins but silencing of each of these members did not affect resistance to $B$. cinerea.}

The RAD23 proteins consist of a small family with four members, AtRAD23a to AtRAD23d, in Arabidopsis (Farmer et al. 2010). To examine whether SISAP4 interacted with other tomato SIRAD23 proteins and whether they had a similar function in immunity against $B$. cinerea, we characterized the tomato SIRAD23 family and identified three additional SIRAD23 members (Solyc03g117780, Solyc04g007120, and Solyc02g085840), in addition to SIRAD23d, by BLASTp searching the tomato genome database using Arabidopsis AtRAD23a to AtRAD23d proteins (Farmer et al. 2010) as queries. Phylogenetic tree analyses showed that the tomato SIRAD23s and Arabidopsis AtRAD23s were clustered into two subgroups (Fig. 8A). Unlike the phylogenetic clustering of two closely related pairs for Arabidopsis AtRAD23s (Farmer et al. 2010), the four tomato SIRAD23s did not form closely related pairs (Fig. 8A). Solyc03g117780, closely related to AtRAD23a and AtRAD23b, was named SIRAD23a, whereas the remaining three SIRAD23 proteins were clustered with the AtRAD23c and AtRAD23d pair. Solyc04g007120, showing high level of similarity to AtRAD23c, was designated as SIRAD23c, whereas Solyc02g063130 (the SISAP4 interactor) and Solyc02g085840 were named SIRAD23d and SIRAD23e, respectively, for convenience in future.

We next examined whether these three SIRAD23s interacted with SISAP4, by $\mathrm{Y} 2 \mathrm{H}$ and BiFC assays. In $\mathrm{Y} 2 \mathrm{H}$ assays, SIRAD23c and SIRAd23e interacted with both the full SISAP4 and the SISAP4-A20 while SIRAD23a did not (Fig. 8B). In BiFC assays, YFP fluorescence was not detected in leaves coinfiltrated with agrobacteria harboring nYFP or cYFP, SIRAD23c-nYFP or cYFP, and SIRAD23e-nYFP or cYFP, whereas significant YFP fluorescence was clearly observed in leaves coinfiltrated with agrobacteria harboring SIRAD23c-nYFP or SISAP4-cYFP and SIRAD23e-nYFP or SISAP4-cYFP (Fig. 8C). Notably again, both the SIRAD23c-SISAP4 and SIRAD23e-SISAP4 interactions were found to occur in both nuclear and cytoplasmic compartments (Fig. 8C). These data indicate that, in addition to interaction with SIRAD23d, SISAP4 also interacted with two additional SIRAD23 proteins, SIRAD23c and SIRAD23e.

To explore the involvement of these three SIRAD23 genes in immunity against $B$. cinerea, we examined the $B$. cinereacaused disease phenotype in the pTRV-SIRAD23a-, pTRVSIRAD23c-, and pTRV-SIRAD23e-infiltrated plants and compared with that in the pTRV-GUS-infiltrated plants. The transcript levels of SIRAD23a, SIRAD23c, and SIRAD23e in the pTRV-SIRAD23a-, pTRV-SIRAD23c-, and pTRV-SIRAD23einfiltrated plants was estimated to be 35 to $40 \%$ of those in pTRV-GUS-infiltrated plants, and silencing of each of 
the SIRAD23 genes did not affect the transcript levels of other SIRAD23 genes. In detached-leaf inoculation assays, the $B$. cinerea-caused disease lesions on leaves of the pTRV-SIRAD23a- and pTRV-SIRAD23c-infiltrated plants were indistinguishable, whereas the lesion on leaves of the pTRV-SIRAD23e-infiltrated plants were slightly smaller than those on leaves of the pTRV-GUS-infiltrated plants (Fig. 8D and E). These data indicate that, unlike SIRAD23d, the other three SIRAD23 members may not be required for immunity against $B$. cinerea, although SIRAD23c and SIRAd23e interact with SISAP4.

\section{SISAP4 and SIRAD23s did not affect immunity} against $P$. syringae pv. tomato $\mathrm{DC} 3000$.

Having proven the positive contributions of SISAP4 and its interactor SIRAD23d to immunity against $B$. cinerea, we asked whether SISAP4 and SIRAD23 proteins play a role in immunity against $P$. syringae pv. tomato DC3000. To this end, we examined the disease phenotype and bacterial growth in leaves of the pTRV-SISAP4- or pTRV-SIRAD23s-infiltrated plants and compared with those in pTRV-GUS-infiltrated plants.
As shown in Figure 9, the pTRV-SISAP4-, pTRV-SIRAD23a-, pTRV-SIRAD23c-, pTRV-SIRAD23d-, and pTRV-SIRAD23einfiltrated plants exhibited similar disease symptom and supported comparable bacterial growth compared with those in the pTRVGUS-infiltrated plants. These data indicate that neither SISAP4 nor SIRAD23 proteins affect immunity against $P$. syringae pv. tomato DC3000 in tomato.

\section{DISCUSSION}

Emerging evidence suggests that SAPs, as either positive or negative regulators, play roles in plant immunity against bacterial pathogens (Kang et al. 2017; Tyagi et al. 2014). In the present study, our VIGS-based functional analyses identified two SISAP genes, SISAP4 and SISAP10, playing roles in tomato immunity against $B$. cinerea. Silencing of SISAP4 resulted in decreased resistance against $B$. cinerea, as the SISAP4-silenced plants exhibited greater disease severity and supported more in-planta growth of the pathogen than the nonsilenced plants (Fig. 2). By contrast, transient expression of SISAP4 in N. benthamiana conferred an increased resistance to B. cinerea (Fig. 3).
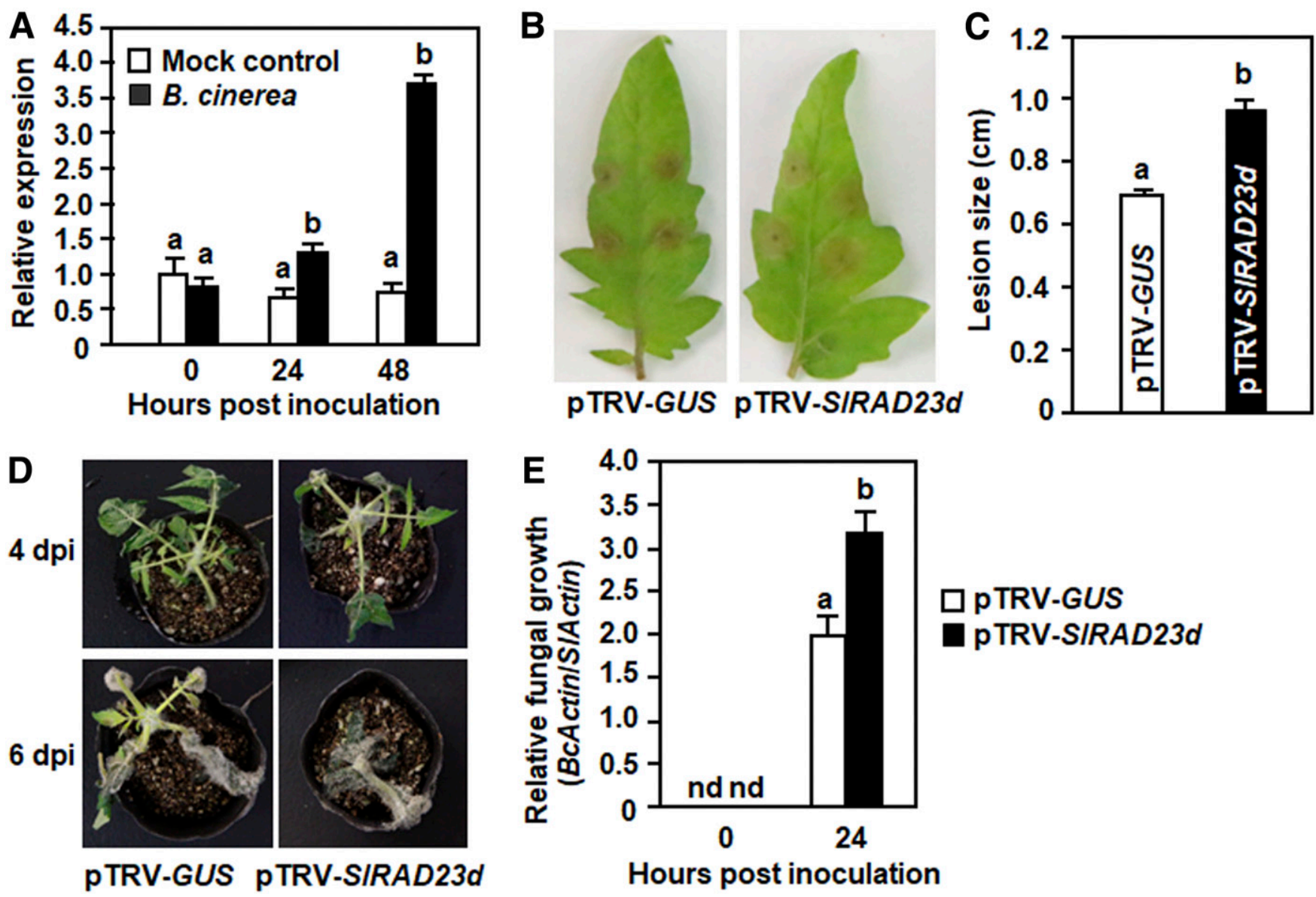

Fig. 7. Silencing of SIRAD23d resulted in decreased resistance to Botrytis cinerea. A, Expression patterns of SIRAD23d in response to infection by $B$. cinerea. Four-week-old plants were inoculated by spore suspension $\left(2 \times 10^{5}\right.$ spores per milliliter $)$ of $B$. cinerea or buffer solution, as a mock control. Leaf samples were collected at indicated timepoints and the transcript level of SIRAD23d was analyzed by quantitative reverse transcription-polymerase chain reaction. Relative expression is shown as fold of the transcript level of the internal SlActin gene. B and $\mathbf{C}$, Representative B. cinerea-caused disease phenotype and lesion size in detached-leaf inoculation assays. D and E, Representative $B$. cinerea disease symptoms and fungal growth in whole-plant inoculation assays. Ten-day-old seedlings were infiltrated with agrobacteria carrying pTRV-SIRAD23d or pTRV-GUS and the virus-induced gene silencing-infiltrated plants were inoculated at 4 weeks after agroinfiltration by dropping spore suspensions $\left(1 \times 10^{5}\right.$ spores per milliliter) of $B$. cinerea on detached leaves or foliar spraying with spore suspension $\left(2 \times 10^{5}\right.$ spores per milliliter) onto leaves of whole plants. Photographs were taken at 3 days postinoculation (dpi) in detached-leaf inoculation assays (B) or at 4 and 6 dpi in whole-plant inoculation assays (D). Lesion size in detached-leaf inoculation assays was measured at 3 dpi (C). In-planta fungal growth in whole-plant inoculation assays was estimated, at the indicated timepoints, by analyzing the transcript levels of B. cinerea BcActin and tomato SlActin as an internal control and are shown as ratios of the transcript level of BcActin to that of SlActin (E). Data presented in C and E are the means \pm standard deviation from three independent experiments and different letters above the columns indicate significant difference at $P<0.05$ level. 
Meanwhile, SISAP4 interacts with SIRAD23d (Fig. 6B), belonging to the RAD23 family that is involved in transferring of ubiquitin conjugates into UPS (Guzder et al. 1998; Shuck et al. 2008) and silencing of SIRAD23d also led to attenuated immunity against $B$. cinerea (Fig. 7). These data demonstrate that SISAP4 acts as a positive regulator of tomato immunity against $B$. cinerea, probably through its interaction with SIRAD23d to transfer substrate targets into UPS for degradation.

It is generally accepted that ROS accumulated during the late infection stage directly benefit the establishment of infection by B. cinerea and that sustained production of ROS as a facilitator of cell death may promote disease development (Mengiste 2012). The facts that the expression of the ROS-generating genes, including SIRBOHI and SlWFIII, was up-regulated while the expression of ROS-scavenging genes such as SISOD and SlCAT was down-regulated in the SlSAP4-silenced plants
(Fig. 4C), leading to the significant accumulation of larger amounts of $\mathrm{H}_{2} \mathrm{O}_{2}$ in the SlSAP4-silenced plants than that in the unsilenced plants at 24 hpi with $B$. cinerea (Fig. 4A and B), indicating an imbalance between ROS generation and scavenging as affected by the reduced function of SISAP4. It is, therefore, likely that accelerated ROS accumulation in B. cinerea-infected SISAP4-silenced plants may be one of the mechanisms leading to attenuated immunity against $B$. cinerea. On the other hand, several biochemical and molecular analyses of the SISAP4-silenced plants before and after pathogen infection suggest that SISAP4 plays its role in immunity against $B$. cinerea through affecting the JA/ET signaling-mediated defense response. Although MeJA did not induce the expression of SISAP4 (Fig. 1B) and expression of SIMYC2, a positive regulator of the JA signaling (Du et al. 2017), in SlSAP4silenced plants was not altered by infection with $B$. cinerea, the JA content was markedly decreased and the expression of

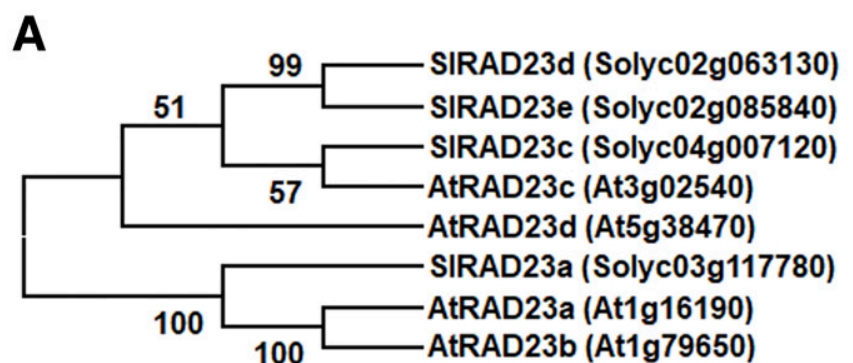

B

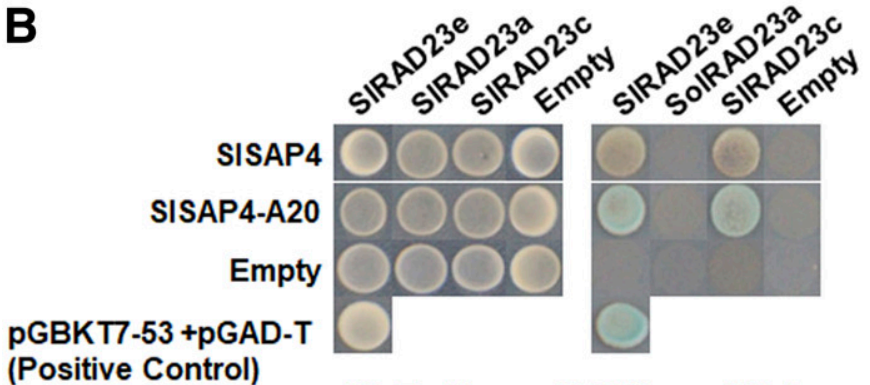

SD-Trp/-Leu QDO/X-a-gal/AbA

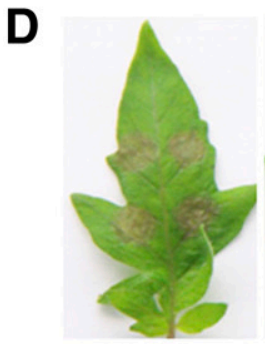

pTRV- GUS

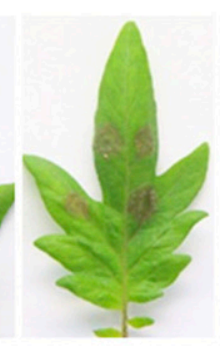

SIRAD23e

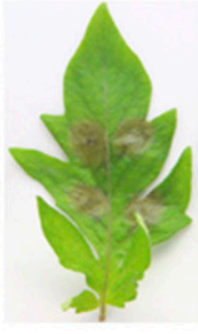

SIRAD23a

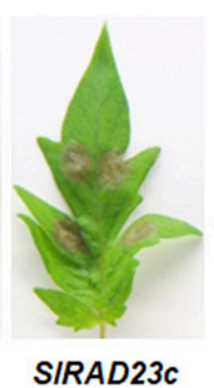

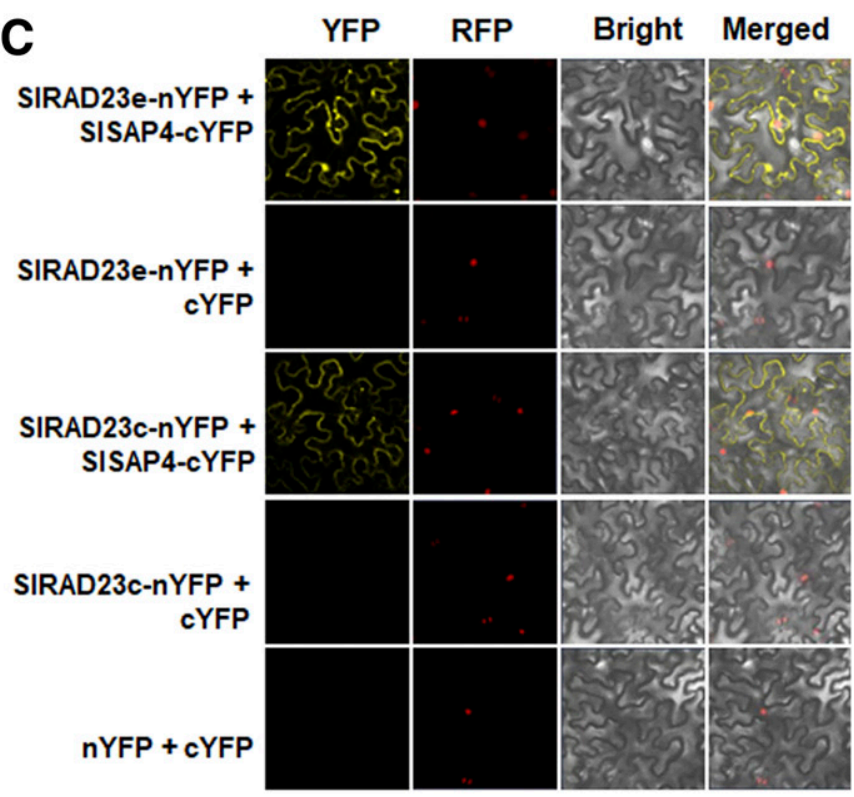

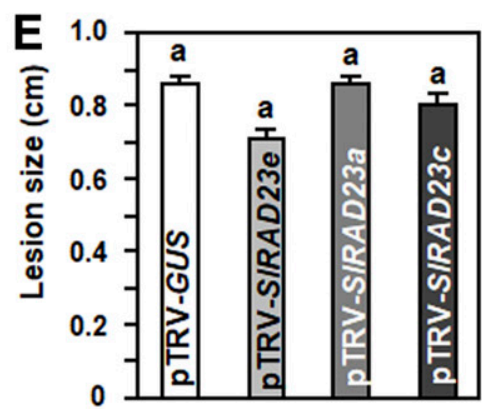

Fig. 8. Interaction of other SIRAD23 family members with SISAP4, but silencing of each of these members did not affect resistance to Botrytis cinerea. A, A phylogenetic tree of SIRAD23s with AtRAD23s. Phylogenetic tree was constructed by the neighbor-joining method, using MEGA program version 6.06, and bootstrap values from 1,000 replicates are indicated at the nodes. B, Interaction of SISAP4 with SIRAD23a, SIRAD23c, or SIRAD23e. The full SISAP4 protein and its A20 domain were fused to the DNA-binding domain in pGBKT7 vector, while SIRAD23a, SIRAD23c, and SIRAD23e were fused to the DNAactivating domain in pGADT7 vector. Yeast cells cotransformed with indicated pairs of vectors were incubated on SD/-Trp/-Leu and SD/-Ade/-His/-Leu/-Trp + $\mathrm{X}-\alpha$-gal/AbA (QDO/X- $\alpha$-gal/AbA) plates to test the interaction activity. Yeast cells cotransformed with pGBKT7 and pGADT7 empty vectors or with pGBKT7-53 and pGADT7-T were used as negative and positive controls, respectively. C, Biomolecular florescence complementation analysis of the interaction of SISAP4 with SIRAD23c and SIRAD23e. Combined mixtures of agrobacteria harboring SIRAD23e-nYFP or SISAP4-cYFP, SIRAD23e-nYFP or cYFP, SIRAD23c-nYFP or SISAP4-cYFP, and SIRAD23c-nYFP or cYFP, and nYFP or cYFP were separately infiltrated into leaves of Nicotiana benthamiana plants and fluorescence was observed under a confocal microscopy at $48 \mathrm{~h}$ after agroinfiltration. D and $\mathbf{E}$, Representative $B$. cinerea-caused disease phenotype and lesion size in detached-leaf-inoculation assays. Ten-day-old seedlings were infiltrated with agrobacteria carrying pTRV-SIRAD23a, pTRV-SIRAD23c, pTRV-SIRAD23e, or pTRV-GUS, and the virus-induced gene silencing-infiltrated plants were inoculated at 4 weeks after agroinfiltration by dropping spore suspension $\left(1 \times 10^{5}\right.$ spores per milliliter) of $B$. cinerea on detached leaves. Photos were taken and lesion sizes were measured at 3 days postinoculation. Data presented in $\mathrm{E}$ are the means \pm standard deviation from three independent experiments and different letters above the columns indicate significant difference at $P<0.05$. 
JA/ET signaling-responsive defense genes was significantly down-regulated in the SISAP4-silenced plants after infection with B. cinerea (Fig. 4E and G). Importantly, exogenous application of MeJA partially restored the resistance of the SISAP4-silenced plants to $B$. cinerea and decreased the B. cinerea-induced ROS accumulation (Fig. 5). Furthermore, the expression of SlSAP4 was induced by ACC (Fig. 1B) and the expression of SIEIL1, a positive regulator of ET signaling
A

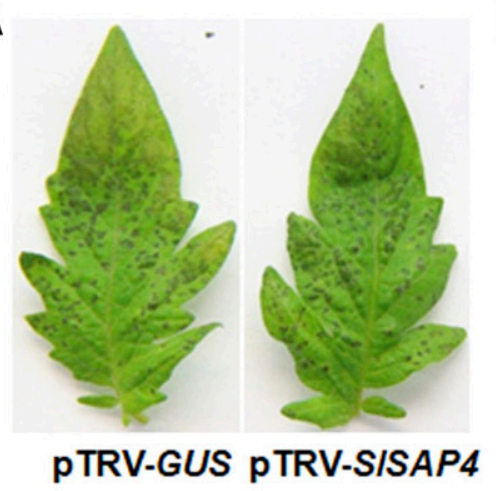

C

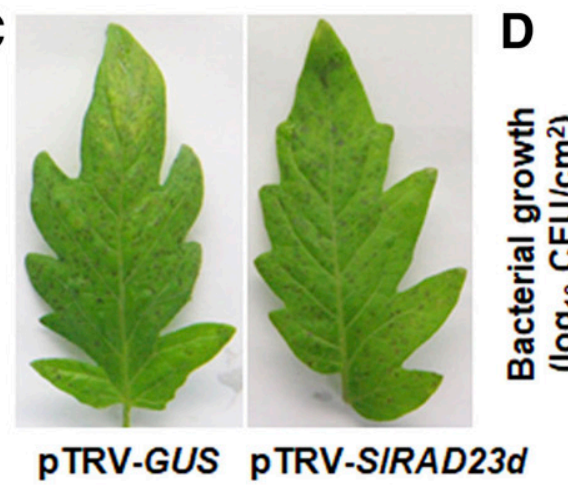

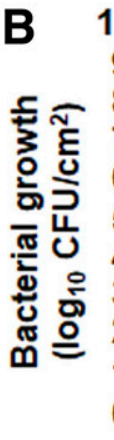
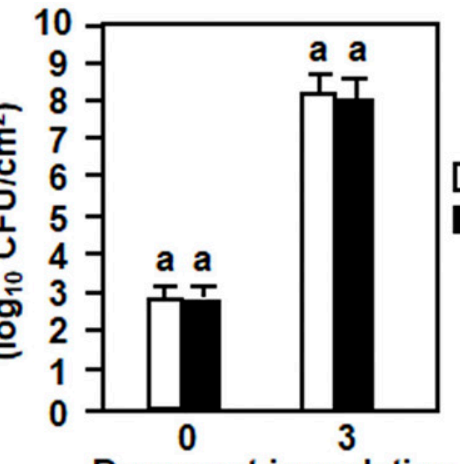

Days post inoculation

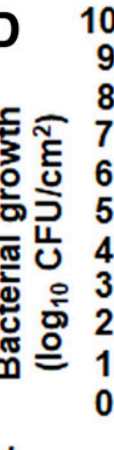

DPTRV-GUS

pTRV-SISAP4

E
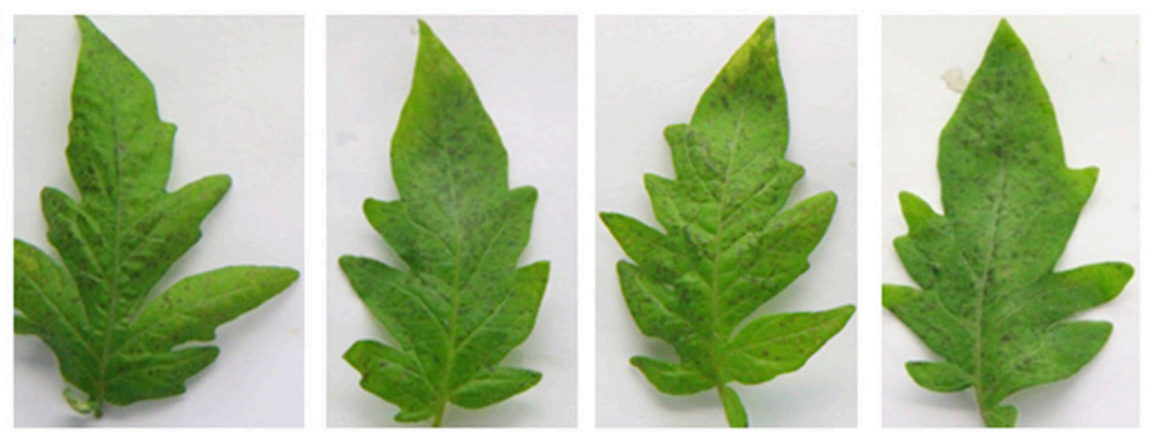

pTRV-GUS

pTRV-SIRAD23e pTRV-SIRAD23a pTRV-SIRAD23c

$\mathbf{F}$

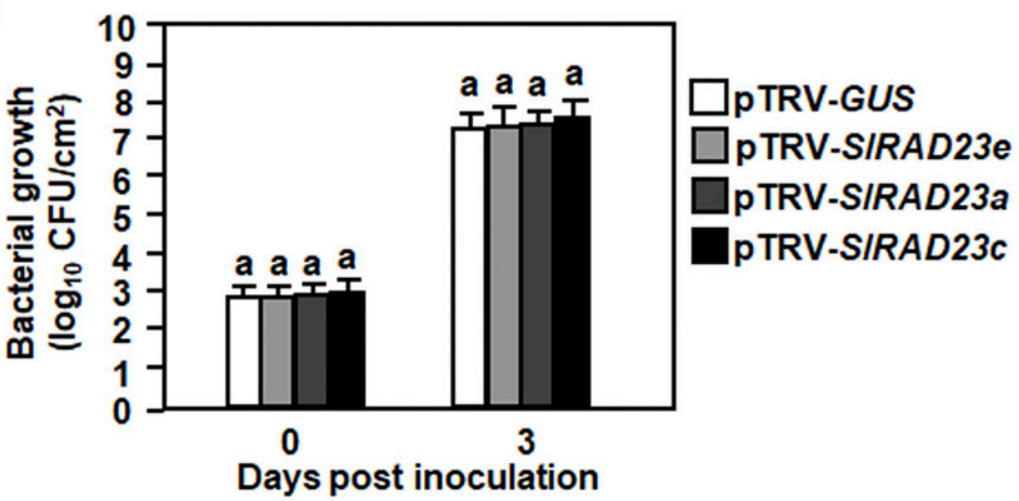

Fig. 9. S1SAP4 and SIRAD23s do not affect the immunity against Pseudomonas syringae pv. tomato DC3000. Ten-day-old seedlings were infiltrated with agrobacteria carrying pTRV-SISAP4, pTRV-RAD23, or pTRV-GUS constructs and the virus-induced gene silencing-infiltrated plants were inoculated, by vacuum infiltration, with $P$. syringae pv. tomato DC3000 (optical density at $600 \mathrm{~nm}=0.0002$ ) at 4 weeks after agroinfiltration. A to F, Representative $P$. syringae DC3000-caused disease symptom (A, C and E) and bacterial growth in inoculated leaves (B, D and F). Photos were taken at 3 days postinoculation (dpi). Leaf samples were collected at 0 and 3 dpi and bacterial titers in leaf tissues was estimated. Data presented in B, D, and F are the means \pm standard deviation from three independent experiments and different letters above the columns indicate significant difference at $P<0.05$. 
(Tieman et al. 2001), was suppressed significantly in the SISAP4-silenced plants after infection with B. cinerea (Fig. 4G). These lines of evidence indicate the involvement of the signaling pathways of JA, ET, or both in SISAP4-mediated immunity against $B$. cinerea. By contrast, SA did not induce the expression of SISAP4 (Fig. 1B) and no alteration in the SA content and the expression of SA signaling defense genes were detected in SISAP4-silenced plants before or after infection by $B$. cinerea (Fig. 4D and F). These facts, together with the fact that silencing of SlSAP4 did not affect immunity against P. syringae pv. tomato DC3000 (Fig. 9A), indicate not only that SA signaling is not involved in the function of SISAP4 immunity against $B$. cinerea but also that SISAP4 does not mediate an antagonistic crosstalk between the JA/ET and SA signaling pathways during its function in immunity against $B$. cinerea.

It was shown that the SAPs can interact with different components in UPS, although interactions of SAPs with other proteins were also identified (Choi et al. 2012; Giri et al. 2011; Kang et al. 2011; Kothari et al. 2016). The Arabidopsis AtSAP5 and Prunus PpSAP1 were found to bind different linkagespecific polyubiquitin chains and, therefore, they exert their biological functions by linking substrate targets for degradation in UPS (Choi et al. 2012; Kang et al. 2011; Lloret et al. 2017). In our $\mathrm{Y} 2 \mathrm{H}$ screening, some ubiquitination-associated proteins with high levels of frequency, e.g., ubiquitin-ribosomal fusion protein (16 times in 44 clones) and ubiquitin (15 times in 44 clones), were identified as SISAP4 interactors. Most importantly, we found that SISAP4 interacted with three of four tomato SIRAD23 proteins, as revealed by $\mathrm{Y} 2 \mathrm{H}, \mathrm{BiFC}$, and co-IP assays (Figs. 6 and 8). This agrees with the observation that Arabidopsis AtSAP5 and AtSAP9 can bind to AtRAD23b and AtRAD23d (Kang et al. 2017). The RAD23 proteins have emerged as shuttles of ubiquitin conjugates by binding ubiquitin-protein conjugates for degradation in UPS (Farmer et al. 2010). The interactions with some ubiquitination-associated proteins imply that the biological function of SISAP4 may be associated with the degradation of substrate targets in UPS.

SAPs contain the characteristic A20 and AN1 domains at their N and C termini (Beyaert et al. 2000; Solanke et al. 2009), whereas RAD23s harbor multiple conserved domains, including one UBL (Ub-like), two UBA (Ub-associated), and one XPC/RAD4 (xeroderma pigmentosum complementation group C/radiation-sensitive 4) (Dantuma et al. 2009; Farmer et al. 2010). These conserved domains in SAP or RAD23 proteins seem to play different roles in interaction with other proteins. The rice OsiSAP1 was also found to interact with OsRLCK253 via the A20 domain for activating stress response (Giri et al. 2011); however, OsSAP1 was shown to interact with an aminotransferase (OsAMTR1) through both A20 and AN1 domains and interact strongly with a pathogenesis-related 1a protein (OsSCP) via the linker region between A20 and AN1 domains (Kothari et al. 2016). In our effort to identify the interacting domains in SISAP4, we found that the A20 domain in SISAP4 is critical for its interaction with SIRAD23d (Fig. 6C). For the interacting domains in SIRAD23d, our deletion analyses revealed that SIRAD23d $\triangle \mathrm{UBL} \triangle \mathrm{UBA} \triangle \mathrm{XPC}$, a deletion mutant harboring only the C-terminal UBA domain, lost the interaction activity with SISAP4 (Fig. 6D), implying that a single UBA domain may be insufficient for its interaction with SISAP4. This result, together with the interaction feature of SIRAD23d $\triangle \mathrm{UBL}$, a deletion mutant harboring the two UBA domains, indicate that both the UBA domains are required for SIRAD23d to interact with SISAP4. Previous studies have shown that the N-terminal UBL of the RAD23 proteins physically interacts with multiple components in UPS while the two UBA domains can bind ubiquitylated proteins, acting as a bridge between UPS and ubiquitylated substrates (Farmer et al.
2010; Kim et al. 2004). However, whether and how SIRAD23d interacts with the components in UPS and thus plays a role in tomato immunity against $B$. cinerea need further investigation.

The RAD23 proteins were first characterized as a DNA repair factor (Guzder et al. 1998; Shuck et al. 2008) and were found to be involved in the transfer of ubiquitinated proteins to UPS (Farmer et al. 2010). The Arabidopsis AtRAD23 proteins appear to play an essential role in cell cycle, morphology, and fertility (Farmer et al. 2010) as well as in UV tolerance (Lahari et al. 2017). Although AtRAD23d and AtRAD23b interact with AtSAP9, which negatively regulates immunity against a nonhost bacterial pathogen (Kang et al. 2017), the function of RAD23 proteins in plant immunity was not yet known. In the present study, we observed that the SIRAD23d-silenced plants exhibited more severe disease and supported more in-planta $B$. cinerea growth than the nonsilenced plants (Fig. 7), indicating a positive contribution of SIRAD23d, like its interacting partner SISAP4, to immunity against B. cinerea. Arabidopsis AtRAD23c and AtRAD23d were found to be required for a phytoplasma effector SAP54enhanced insect vector colonization, indicating an involvement of RAD23 proteins in plant defense to insects (MacLean et al. 2014). However, silencing of each of the other three SIRAD23 genes did not affect immunity against B. cinerea, although SIRAD23c and SIRAD23e also interacted with SISAP4 (Fig. 8). On the other hand, silencing of either SISAP4 or each of the four SIRAD23 genes caused no significant change in immunity against $P$. syringae pv. tomato DC3000 (Fig. 9), indicating that S1SAP4 and SIRAD23d as well as their physical interaction may play specific roles in immunity against $B$. cinerea.

The biochemical characteristics of SISAP4 observed in the present study differ from those of some previously reported plant SAP proteins. Similar to the animal A20 protein acting as a ubiquitin E3 ligase (Heyninck and Beyaert 2005), Arabidopsis AtSAP5 and AtSAP9 and rice OsSAP7 have been shown to possess ubiquitin E3 ligase activity in vitro and ubiquitinate their substrates (Kang et al. 2011, 2013, 2017; Sharma et al. 2015). In our repeated experiments, however, we were unable to detect the ubiquitin E3 ligase activity of SISAP4 in vitro, while the known ubiquitin E3 ligase AtPUB13 (Liao et al. 2017) showed a significant activity to catalyze the formation of ubiquitin conjugates (Fig. 6A). Although Arabidopsis AtSAP9 has ubiquitin E3 ligase activity and interacts with AtRAD23d, no ubiquitination of AtRAD23d by AtSAP9 was detected (Kang et al. 2017). It is likely that SISAP4 may exert its biological function without ubiquitin E3 ligase activity but interacts with the ubiquitin-proteasome system by another mechanism. On the other hand, it was reported that the interaction between Arabidopsis AtSAP9 and AtRAD23d occurred in nucleus (Kang et al. 2017) and the AtRAD23 proteins were nucleus enriched with weak distribution in cytoplasmic compartment (Farmer et al. 2010). By contrast, SISAP4 and its interactor SIRAD23d seem not to localize solely in nucleus, and their localization and interaction occur in both cytoplasmic and nuclear compartments (Fig. 6B). The interaction of OsSAP1 and its partners was also found to occur in whole cells but was not restricted solely in nucleus (Kothari et al. 2016).

The involvement of plant SAPs in growth and development has been reported recently. We observed in this study that the SISAP10-silenced plants displayed an earlier accelerated yellowing phenotype accompanying reduced chlorophyll content in leaves, indicating that SISAP10 may be involved in maintenance of the integrity of chlorophyll. This is similar to the observation that overexpression of Medicago truncatula $M t S A P 1$ led to higher chlorophyll content in transgenic 
tobacco plants (Charrier et al. 2012). Silencing of each of other 12 SISAP genes examined did not cause any defect in growth and development; however, the involvement of these SISAPs in tomato growth and development cannot be ruled out. It was recently found that overexpression of either OsSAP11 or ZFP185 results in a dwarfism or semidwarfism phenotype through negatively regulating cell elongation or cell size, respectively (Liu et al. 2011; Zhang et al. 2016).

The expression of some SISAP genes, including SISAP1, SISAP3, SlSAP4, SISAP6, SlSAP7, SlSAP11, and SlSAP12, was significantly upregulated in response to $B$. cinerea (Fig. 1A). VIGS-based functional analyses revealed that only silencing of SISAP4 or SISAP1O resulted in increased susceptibility to $B$. cinerea, whereas silencing of each of the other highly induced SISAP genes did not lead to a significant change in resistance to $B$. cinerea (Fig. 2). It seems likely that most of these highly induced SlSAP genes are not required for immunity against $B$. cinerea. In our study regarding the function of SISAP3, one of these highly induced SISAP genes, we found that silencing of SISAP3 did not alter resistance to $B$. cinerea, while overexpression in stable transgenic tomato conferred an increased resistance against $B$. cinerea (S. Liu and F. Song, unpublished data). Therefore, the involvement of these highly induced SISAP genes in immunity against $B$. cinerea cannot be ruled out before functional analyses with transient and stable overexpression experiments are performed.

In summary, we demonstrate that SISAP4 contributes positively to immunity against $B$. cinerea through affecting JA/ET signaling and interacts with SIRAD23d for degradation of the substrate targets in UPS. Further biochemical and molecular investigations are required to identify the substrate targets that are transferred into UPS for degradation by the SISAP4-SIRAD23d interaction event and to examine the involvement of SISAP4 and SIRAD23s in immunity using overexpression approach.

\section{MATERIALS AND METHODS}

\section{Plant cultivation and hormone treatment.}

Tomato (Solanum lycopersicum) 'Suhong 2003' was used for most of the experiments while 'MicroTom' was used for wholeplant inoculation assays. Tomato growth, hormone treatment, pathogen inoculation, disease evaluation, and measurement of in-planta pathogen growth were carried out as described previously (AbuQamar et al. 2008; Li et al. 2014; Zhang et al. 2014, 2015). In B. cinerea disease assays, the spore density in suspension was adjusted to $1 \times 10^{5}$ spores per milliliter for detached-leaf inoculation assays or to $2 \times 10^{5}$ spores per milliliter for whole plant inoculation assays.

\section{Construction of vectors and VIGS assays.}

VIGS fragments of SISAP and SIRAD23 genes were amplified using gene-specific primers (Supplementary Table S1) and were cloned into the pTRV vector (Liu et al. 2002), yielding plasmids pTRV-SISAPs or pTRV-SIRAD23s. Sequence information for the VIGS fragments is listed in Supplementary Table S2. Before VIGS assays, agrobacteria harboring pTRVSISAPs, pTRV-SIRAD23s, or pTRV-GUS (a negative control plasmid with insertion of a GUS fragment) were mixed with agrobacteria carrying TRV1 in a ratio of $1: 1$, and agroinfiltration was then performed by infiltrating the mixed agrobacterial suspension into the abaxial surface of 10-day-old seedlings, using 1-ml needleless syringes. Similar procedures with agrobacteria carrying pTRV-SIPDS as a marker of silencing was included in each of the VIGS experiments to evaluate the efficiency of the VIGS protocol. Once the
pTRV-SIPDS-infiltrated plant bleaching phenotype appeared around 10 days postagroinfiltraton, leaf samples were collected, at 14 days postagroinfiltration, from the pTRV-SISAPs-, pTRV-SIRAD23s-, or pTRV-GUS-infiltrated plants to estimate the silencing efficiency by qRT-PCR. The pTRV-SISAPs-, pTRV2-SIRAD23s-, or pTRV2-GUS-infiltrated plants with significant silencing of the target gene were used for disease assays and other experiments at 4 weeks after agroinfiltration.

\section{Transient expression of SlSAP4 in N. benthamiana.}

The coding sequence of SISAP4 was amplified with genespecific primers, was sequenced, and was cloned into pFGCEgfp vector to make the S1SAP4-GFP fusion construct pFGC-Egfp-SISAP4-GFP. Agrobacteria carrying pFGC-EgfpSISAP4-GFP or pFGC-Egfp were infiltrated into fully expanded leaves of 4-week-old $N$. benthamiana plants, as described previously ( $\mathrm{Li}$ et al. 2014), and leaf samples were collected for qRT-PCR analysis of SISAP4 expression, Western blotting of GFP, and a detached-leaf assay of disease phenotype.

\section{Purification of recombinant SISAP4 protein and in vitro ubiquitination assay.}

The coding sequence of SISAP4 was amplified with genespecific primers and was cloned into pET32a vector, yielding pET32a-SISAP4, to make the His-SISAP4 fusion protein, and was then was introduced into the E. coli Rosetta strain (Novagen, San Diego, CA, U.S.A.). Induction and purification of recombinant His-SISAP4 protein in E. coli cells were performed using Ni-NTA-agarose (QiaGen, Valencia, CA, U.S.A.) according to the manufacturer's instructions. The purified His-SISAP4 protein was analyzed by sodium dodecyl sulfate-polyacrylamide gel electrophoresis (SDSPAGE) and immunoblotting with $6 \times$ His antibody. In-vitro ubiquitination assay was performed with $5 \mu \mathrm{g}$ of ubiquitin (Boston Biochem, Cambridge, MA, U.S.A.), $110 \mathrm{ng}$ of E1 (Millipore, Darmstadt, Germany), $100 \mathrm{ng}$ of human recombinant UbcH2 (Abcam, Cambridge, U.K.), and was purified with $4 \mu \mathrm{g}$ of His-SISAP4 in reaction buffer $(20 \mathrm{mM}$ MOPS, pH 7.2, $100 \mathrm{mM} \mathrm{KCl,} 5 \mathrm{mM} \mathrm{MgCl}_{2}, 5 \mathrm{mM}$ ATP, and $10 \mathrm{mM}$ dithiothreitol). The reaction mixture was incubated for $3 \mathrm{~h}$ at $30^{\circ} \mathrm{C}$, was stopped by adding SDS-PAGE loading buffer and boiling for $5 \mathrm{~min}$, followed by separation on a $12 \%$ SDSPAGE. Immunoblotting was performed using an anti-Ub antibody (CalBiochem, La Jolla, CA, U.S.A.), and chemiluminescence signals were detected with SuperSignal West Pico PLUS chemiluminescent substrate kit (Thermo Fisher Scientific, Waltham, MA, U.S.A.) according to the manufacture's recommendations.

\section{Y2H screening and verification of SISAP4 interactors and determination of the interaction domains in SISAP4 and SIRAD23d.}

Complementary DNA was synthesized with total RNA extracted from $B$. cinerea-infected leaf samples. Generation of a tomato Y2H library and screening for SISAP4 interactors were performed using Matchmaker library construction and screening kit (Clontech Laboratories, Mountain View, CA, U.S.A.) according to the manufacturer's instructions. The coding sequence of SISAP4 was amplified with gene-specific primers and was cloned into pGBKT7 vector, yielding pGBKT7-SISAP4 plasmid, and was then transformed into the $\mathrm{Y} 2 \mathrm{H}$ library yeast cells. The transformants were selected on SD/-Leu/-Trp media containing $40 \mu \mathrm{g}$ of $\mathrm{X}-\alpha-\mathrm{Gal}$ per milliliter and $125 \mathrm{ng}$ of Aureobasidin A per milliliter. The activating domain plasmids were rescued from putative positive clones and were sequenced. For detecting the interaction between SISAP4 and SIRAD23s, 
the coding sequences of SIRAD23 genes were amplified with gene-specific primers, were sequenced and cloned in-frame into pGADT7-Rec plasmid, and were transformed into yeast cells harboring pBGKT7-SISAP4 plasmid. For determining the domains responsible for interaction in SISAP4 and SIRAD23d, different deletion mutants (SISAP4-A20, SISAP4-AN1-BD, and S1SAP4 $\triangle \mathrm{A} 20 \triangle \mathrm{AN} 1$ ) of SISAP4 and deletion mutants of SIRAD23d (SIRAD23d $\triangle U B L, \quad S I R A D 23 d \Delta U B L \Delta U B A$, and SIRAD23d $\Delta$ UBL $\Delta U B A \Delta X P C)$ were generated by PCR amplification with gene-specific primers and were cloned in-frame into pGBKT7 or pGADT7-Rec plasmids, respectively. Combined pairs of recombinant pGBKT7 plasmids containing SISAP4 or its deletion mutants and pGADT7-Rec plasmids harboring SIRAD23s or SIRAD23d deletion mutants were cotransformed into yeast cells and the interaction activity was examined by plating yeast cells on SD/-Leu/-Trp and SD/Ade/-His/-Leu/-Trp/ X- $a$-Gal/AbA media.

\section{BiFC assays.}

BiFC assays for determining the interaction between SISAP4 and SIRAD23s were performed as described previously (Yang et al. 2007). The coding sequences of SISAP4 and SIRAD23s were amplified with gene-specific primers, were sequenced, and were cloned into $\mathrm{p} 2 \mathrm{YN}$ or $\mathrm{p} 2 \mathrm{YC}$ plasmids, respectively, fusing in-frame at the C-terminals to nYFP or cYFP and yielding plasmids p2YN-SISAP4, p2YN-SIRAD23c, p2YN-SIRAD23e, p2YC-SISAP4, and p2YC-SIRAD23d. Agrobacteria harboring different recombinant $\mathrm{p} 2 \mathrm{YN}$ or $\mathrm{p} 2 \mathrm{YC}$ plasmids, empty plasmids, or P19 antisilencing factor plasmid were mixed in a ratio of $1: 1: 1(\mathrm{vol} / \mathrm{vol} / \mathrm{vol})$ and were then infiltrated into the abaxial air spaces of leaves of 2-week-old $N$. benthamiana plants. Leaf samples were collected two days after agroinfiltration and fluorescence was observed under a Zeiss LSM780 confocal laser scanning microscope (Zeiss, Oberkochen, Germany).

\section{Measurement of SA and JA contents and in situ detection of $\mathrm{H}_{2} \mathrm{O}_{2}$.}

Extraction of SA and JA from leaf tissues was carried out according to a previously described protocol (Hong et al. 2017) and measurement of SA and JA was performed by a highperformance liquid chromatography-triple quadrupole liquid chromatography-mass spectrometry system (model 1290/6460; Agilent Technologies, Santa Clara, CA, U.S.A.) with stable isotope-labeled SA and JA as standards (Fu et al. 2012). In situ detection of $\mathrm{H}_{2} \mathrm{O}_{2}$ was carried out by DAB staining (ThordalChristensen et al. 1997). Leaf samples were collected from B. cinerea- and mock-inoculated plants at $24 \mathrm{hpi}$ and were incubated in DAB (Sigma-Aldrich, St. Louis) solution $(1 \mathrm{mg} / \mathrm{ml}, \mathrm{pH} 3.8)$ for $8 \mathrm{~h}$ in the dark at room temperature. Accumulation of $\mathrm{H}_{2} \mathrm{O}_{2}$ was visualized using a digital camera and was quantified using ImageJ software (National Institutes of Health) from DAB image (Li et al. 2015).

\section{qRT-PCR analysis of gene expression.}

Total RNA was extracted using Trizol reagent (TaKaRa, Dalian, China) and was treated with RNase-free DNase to erase genomic DNA, according to the manufacturer's instructions. First-strand cDNA was synthesized from $1 \mu \mathrm{g}$ of total RNA by reverse transcription, using the PrimeScriptRT reagent kit (TaKaRa) according to the manufacturer's protocol. qPCR was performed on a CFX96 real-time PCR system (BioRad, Hercules, CA, U.S.A.), and each reaction contained $12.5 \mu \mathrm{l}$ of SYBR PremixEx Taq (TaKaRa), $0.1 \mu \mathrm{g}$ of cDNA, and $7.5 \mathrm{pmol}$ of each gene-specific primer in a final volume of $25 \mu \mathrm{l}$. A tomato SlActin gene (accession number AB199316) was used as an internal control to normalize the qRT-PCR data and relative expression levels of genes of interest were calculated using the $2^{-\Delta \Delta}$ cycle threshold method. For expression patterns of SISAP genes in response to $B$. cinerea and defense signaling hormones, relative expression is presented in a heatmap using fold change values transformed to $\log _{2}$ format by MeV4.9 software.

\section{Statistical analysis.}

All experiments described were performed in triplicate. Data obtained were analyzed statistically according to the Student's $t$ test and the probability values of $P<0.05$ were considered as significant difference.

\section{ACKNOWLEDGMENTS}

We thank D. M. Bisaro (Department of Molecular Genetics, Ohio State University) for providing the BiFC plasmids, M. Goodin (Department of Plant Pathology, University of Kentucky) for providing the H2B-RFP $N$. benthamiana line, and Y. Liang (Institute of Biotechnology, Zhejiang University) for providing the purified recombinant AtPUB13 protein.

\section{LITERATURE CITED}

AbuQamar, S., Chai, M. F., Luo, H., Song, F., and Mengiste, T. 2008. Tomato protein kinase $1 \mathrm{~b}$ mediates signaling of plant responses to necrotrophic fungi and insect herbivory. Plant Cell 20:1964-1983.

AbuQamar, S., Moustafa, K., and Tran, L. S. 2017. Mechanisms and strategies of plant defense against Botrytis cinerea. Crit. Rev. Biotechnol. 37:262-274.

Beyaert, R., Heyninck, K., and Van Huffel, S. 2000. A20 and A20-binding proteins as cellular inhibitors of nuclear factor-kappa B-dependent gene expression and apoptosis. Biochem. Pharmacol. 60:1143-1151.

Bigeard, J., Colcombet, J., and Hirt, H. 2015. Signaling mechanisms in pattern-triggered immunity (PTI). Mol. Plant 8:521-539.

Boller, T., and He, S. Y. 2009. Innate immunity in plants: An arms race between pattern recognition receptors in plants and effectors in microbial pathogens. Science 324:742-744.

Broekaert, W. F., Delauré, S. L., De Bolle, M. F., and Cammue, B. P. 2006. The role of ethylene in host-pathogen interactions. Annu. Rev. Phytopathol. 44:393-416

Chakrabarty, R., Banerjee, R., Chung, S. M., Farman, M., Citovsky, V., Hogenhout, S. A., Tzfira, T., and Goodin, M. 2007. PSITE vectors for stable integration or transient expression of autofluorescent protein fusions in plants: Probing Nicotiana benthamiana-virus interactions. Mol. Plant-Microbe Interact. 20:740-750.

Chang, L., Chang, H. H., Chang, J. C., Lu, H. C., Wang, T. T., Hsu, D. W., Tzean, Y., Cheng, A. P., Chiu, Y. S., and Yeh, H. H. 2018. lant A20/AN1 protein serves as the important hub to mediate antiviral immunity. PLoS Pathog. 14:e1007288.

Charrier, A., Planchet, E., Cerveau, D., Gimeno-Gilles, C., Verdu, I., Limami, A. M., and Lelièvre, E. 2012. Overexpression of a Medicago truncatula stress-associated protein gene (MtSAP1) leads to nitric oxide accumulation and confers osmotic and salt stress tolerance in transgenic tobacco. Planta 236:567-577.

Choi, H., Han, S., Shin, D., and Lee, S. 2012. Polyubiquitin recognition by AtSAP5, an A20-type zinc finger containing protein from Arabidopsis thaliana. Biochem. Biophys. Res. Commun. 419:436-440.

Cui, H., Tsuda, K., and Parker, J. E. 2015. Effector-triggered immunity: From pathogen perception to robust defense. Annu. Rev. Plant Biol. 66:487-511

Dantuma, N. P., Heinen, C., and Hoogstraten, D. 2009. The ubiquitin receptor $\operatorname{Rad} 23$ : At the crossroads of nucleotide excision repair and proteasomal degradation. DNA Repair (Amst.) 8:449-460.

Dean, R., Van Kan, J. A., Pretorius, Z. A., Hammond-Kosack, K. E., Di Pietro, A., Spanu, P. D., Rudd, J. J., Dickman, M., Kahmann, R., Ellis, J., and Foster, G. D. 2012. The top 10 fungal pathogens in molecular plant pathology. Mol. Plant Pathol. 13:414-430.

Derksen, H., Rampitsch, C., and Daayf, F. 2013. Signaling crosstalk in plant disease resistance. Plant Sci. 207:79-87.

Du, M., Zhao, J., Tzeng, D. T. W., Liu, Y., Deng, L., Yang, T., Zhai, Q., Wu, F., Huang, Z., Zhou, M., Wang, Q., Chen, Q., Zhong, S., Li, C. B., and Li, C. 2017. MYC2 orchestrates a hierarchical transcriptional cascade that regulates jasmonate-mediated plant immunity in tomato. Plant Cell 29: 1883-1906.

Farmer, L. M., Book, A. J., Lee, K. H., Lin, Y. L., Fu, H., and Vierstra, R. D. 2010. The RAD23 family provides an essential connection between the 26S proteasome and ubiquitylated proteins in Arabidopsis. Plant Cell 22: 124-142. 
Fu, J., Chu, J., Sun, X., Wang, J., and Yan, C. 2012. Simple, rapid, and simultaneous assay of multiple carboxyl containing phytohormones in wounded tomatoes by UPLC-MS/MS using single SPE purification and isotope dilution. Anal. Sci. 28:1081-1087.

Giri, J., Dansana, P. K., Kothari, K. S., Sharma, G., Vij, S., and Tyagi, A. K. 2013. SAPs as novel regulators of abiotic stress response in plants. BioEssays 35:639-648.

Giri, J., Vij, S., Dansana, P. K., and Tyagi, A. K. 2011. Rice A20/AN1 zinc-finger containing stress-associated proteins (SAP1/11) and a receptor-like cytoplasmic kinase (OsRLCK253) interact via A20 zinc-finger and confer abiotic stress tolerance in transgenic Arabidopsis plants. New Phytol. 191:721-732.

Glazebrook, J. 2005. Contrasting mechanisms of defense against biotrophic and necrotrophic pathogens. Annu. Rev. Phytopathol. 43:205-227.

Guzder, S. N., Sung, P., Prakash, L., and Prakash, S. 1998. Affinity of yeast nucleotide excision repair factor 2, consisting of the Rad4 and $\operatorname{Rad} 23$ proteins, for ultraviolet damaged DNA. J. Biol. Chem. 273: 31541-31546.

Heyninck, K., and Beyaert, R. 2005. A20 inhibits NF-кB activation by dual ubiquitin-editing functions. Trends Biochem. Sci. 30:1-4.

Hong, Y., Yang, Y., Zhang, H., Huang, L., Li, D., and Song, F. 2017. Overexpression of MoSM1, encoding for an immunity-inducing protein from Magnaporthe oryzae, in rice confers broad-spectrum resistance against fungal and bacterial diseases. Sci. Rep. 7:41037.

Jones, J. D., and Dangl, J. L. 2006. The plant immune system. Nature 444: 323-329.

Kang, M., Abdelmageed, H., Lee, S., Reichert, A., Mysore, K. S., and Allen, R. D. 2013. AtMBP-1, an alternative translation product of LOS2, affects abscisic acid responses and is modulated by the E3 ubiquitin ligase AtSAP5. Plant J. 76:481-493.

Kang, M., Fokar, M., Abdelmageed, H., and Allen, R. D. 2011. Arabidopsis SAP5 functions as a positive regulator of stress responses and exhibits E3 ubiquitin ligase activity. Plant Mol. Biol. 75:451-466.

Kang, M., Lee, S., Abdelmageed, H., Reichert, A., Lee, H. K., Fokar, M., Mysore, K. S., and Allen, R. D. 2017. Arabidopsis stress associated protein 9 mediates biotic and abiotic stress responsive ABA signaling via the proteasome pathway. Plant Cell Environ. 40:702-716.

Kim, I., Mi, K., and Rao, H. 2004. Multiple interactions of rad23 suggest a mechanism for ubiquitylated substrate delivery important in proteolysis. Mol. Biol. Cell 15:3357-3365.

Kothari, K. S., Dansana, P. K., Giri, J., and Tyagi, A. K. 2016. Rice stress associated protein 1 (OsSAP1) interacts with aminotransferase (OsAMTR1) and pathogenesis-related 1a protein (OsSCP) and regulates abiotic stress responses. Front. Plant Sci. 7:1057.

Lahari, T., Lazaro, J., and Schroeder, D. F. 2017. RAD4 and RAD23/HMR contribute to Arabidopsis UV tolerance. Genes (Basel) 9:8.

Lai, Z., and Mengiste, T. 2013. Genetic and cellular mechanisms regulating plant responses to necrotrophic pathogens. Curr. Opin. Plant Biol. 16:505-512.

Li, B., Meng, X., Shan, L., and He, P. 2016. Transcriptional regulation of pattern-triggered immunity in plants. Cell Host Microbe 19:641-650.

Li, D., Zhang, H., Song, Q., Wang, L., Liu, S., Hong, Y., Huang, L., and Song, F. 2015. Tomato Sl3-MMP, a member of the Matrix metalloproteinase family, is required for disease resistance against Botrytis cinerea and Pseudomonas syringae pv. tomato DC3000. BMC Plant Biol. 15:143.

Li, X., Zhang, Y., Huang, L., Ouyang, Z., Hong, Y., Zhang, H., Li, D., and Song, F. 2014. Tomato SIMKK2 and SIMKK4 contribute to disease resistance against Botrytis cinerea. BMC Plant Biol. 14:166.

Liao, D., Cao, Y., Sun, X., Espinoza, C., Nguyen, C. T., Liang, Y., and Stacey, G. 2017. Arabidopsis E3 ubiquitin ligase PLANT U-BOX13 (PUB13) regulates chitin receptor LYSIN MOTIF RECEPTOR KINASE5 (LYK5) protein abundance. New Phytol. 214:1646-1656.

Liu, Y., Schiff, M., and Dinesh-Kumar, S. P. 2002. Virus-induced gene silencing in tomato. Plant J. 31:777-786.

Liu, Y., Xu, Y., Xiao, J., Ma, Q., Li, D., Xue, Z., and Chong, K. 2011. OsDOG, a gibberellin-induced A20/AN1 zinc-finger protein, negatively regulates gibberellin-mediated cell elongation in rice. J. Plant Physiol. 168: 1098-1105.

Lloret, A., Conejero, A., Leida, C., Petri, C., Gil-Muñoz, F., Burgos, L., Badenes, M. L., and Ríos, G. 2017. Dual regulation of water retention and cell growth by a stress-associated protein (SAP) gene in Prunus. Sci. Rep. 7:332.

MacLean, A. M., Orlovskis, Z., Kowitwanich, K., Zdziarska, A. M., Angenent, G. C., Immink, R. G., and Hogenhout, S. A. 2014 Phytoplasma effector SAP54 hijacks plant reproduction by degrading MADS-box proteins and promotes insect colonization in a RAD23dependent manner. PLoS Biol. 12:e1001835.

Mengiste, T. 2012. Plant immunity to necrotrophs. Annu. Rev. Phytopathol. 50:267-294.
Peng, Y., van Wersch, R., and Zhang, Y. 2018. Convergent and divergent signaling in PAMP-triggered immunity and effector-triggered immunity. Mol. Plant-Microbe Interact. 31:403-409.

Pieterse, C. M., Van der Does, D., Zamioudis, C., Leon-Reyes, A., and Van Wees, S. C. 2012. Hormonal modulation of plant immunity. Annu. Rev. Cell Dev. Biol. 28:489-521.

Pieterse, C. M. J., Leon-Reyes, A., Van der Ent, S., and Van Wees, S. C. 2009. Networking by small-molecule hormones in plant immunity. Nat. Chem. Biol. 5:308-316.

Schwessinger, B., and Ronald, P. C. 2012. Plant innate immunity: Perception of conserved microbial signatures. Annu. Rev. Plant Biol. 63:451-482.

Segonzac, C., and Zipfel, C. 2011. Activation of plant pattern-recognition receptors by bacteria. Curr. Opin. Microbiol. 14:54-61.

Sharma, G., Giri, J., and Tyagi, A. K. 2015. Rice OsiSAP7 negatively regulates ABA stress signalling and imparts sensitivity to water-deficit stress in Arabidopsis. Plant Sci. 237:80-92.

Shigenaga, A. M., and Argueso, C. T. 2016. No hormone to rule them all: Interactions of plant hormones during the responses of plants to pathogens. Semin. Cell Dev. Biol. 56:174-189.

Shuck, S. C., Short, E. A., and Turchi, J. J. 2008. Eukaryotic nucleotide excision repair: From understanding mechanisms to influencing biology Cell Res. 18:64-72.

Solanke, A. U., Sharma, M. K., Tyagi, A. K., and Sharma, A. K. 2009. Characterization and phylogenetic analysis of environmental stressresponsive SAP gene family encoding A20/AN1 zinc finger proteins in tomato. Mol. Genet. Genomics 282:153-164.

Spoel, S. H., and Dong, X. 2012. How do plants achieve immunity? Defence without specialized immune cells. Nat. Rev. Immunol. 12:89-100.

Ströher, E., Wang, X. J., Roloff, N., Klein, P., Husemann, A., and Dietz, K. J. 2009. Redox-dependent regulation of the stress-induced zinc-finger protein SAP12 in Arabidopsis thaliana. Mol. Plant 2:357-367.

Thordal-Christensen, H., Zhang, Z. G., Wei, Y. D., and Collinge, D. B. 1997. Subcellular localization of $\mathrm{H}_{2} \mathrm{O}_{2}$ in plants. $\mathrm{H}_{2} \mathrm{O}_{2}$ accumulation in papillae and hypersensitive response during the barley-powdery mildew interaction. Plant J. 11:1187-1194.

Tieman, D. M., Ciardi, J. A., Taylor, M. G., and Klee, H. J. 2001. Members of the tomato LeEIL (EIN3-like) gene family are functionally redundant and regulate ethylene responses throughout plant development. Plant J. 26:47-58.

Tsuda, K., and Somssich, I. E. 2015. Transcriptional networks in plant immunity. New Phytol. 206:932-947.

Tyagi, H., Jha, S., Sharma, M., Giri, J., and Tyagi, A. K. 2014. Rice SAPs are responsive to multiple biotic stresses and overexpression of OsSAP1, an A20/AN1 zinc-finger protein, enhances the basal resistance against pathogen infection in tobacco. Plant Sci. 225:68-76.

Vij, S., and Tyagi, A. K. 2006. Genome-wide analysis of the stress associated protein (SAP) gene family containing A20/AN1 zinc-finger(s) in rice and their phylogenetic relationship with Arabidopsis. Mol. Genet. Genomics 276:565-575

Wang, X., Jiang, N., Liu, J., Liu, W., and Wang, G. L. 2014. The role of effectors and host immunity in plant-necrotrophic fungal interactions. Virulence 5:722-732.

Yang, X., Baliji, S., Buchmann, R. C., Wang, H., Lindbo, J. A., Sunter, G., and Bisaro, D. M. 2007. Functional modulation of the geminivirus AL2 transcription factor and silencing suppressor by self-interaction. J. Virol. 81:11972-11981.

Zhang, J., and Zhou, J. M. 2010. Plant immunity triggered by microbial molecular signatures. Mol. Plant 3:783-793.

Zhang, L., Zhang, F., Melotto, M., Yao, J., and He, S. Y. 2017. Jasmonate signaling and manipulation by pathogens and insects. J. Exp. Bot. 68: 1371-1385.

Zhang, Y., Jin, X., Ouyang, Z., Li, X., Liu, B., Huang, L., Hong, Y., Zhang, H., Song, F., and Li, D. 2015. Vitamin B6 contributes to disease resistance against Pseudomonas syringae pv. tomato DC3000 and Botrytis cinerea in Arabidopsis thaliana. J. Plant Physiol. 175:21-25.

Zhang, Y., Lan, H., Shao, Q., Wang, R., Chen, H., Tang, H., Zhang, H., and Huang, J. 2016. An A20/AN1-type zinc finger protein modulates gibberellins and abscisic acid contents and increases sensitivity to abiotic stress in rice (Oryza sativa). J. Exp. Bot. 67:315-326.

Zhang, Y., Liu, B., Li, X., Ouyang, Z., Huang, L., Hong, Y., Zhang, H., Li, D., and Song, F. 2014. The de novo biosynthesis of vitamin B6 is required for disease resistance against Botrytis cinerea in tomato. Mol. Plant-Microbe Interact. 27:688-699.

\section{AUTHOR-RECOMMENDED INTERNET RESOURCE}

Center for Cancer Computational Biology, MeV4.9 software: http://www.tm4.org 